\title{
Ultra-fast heat dissipating aerogels derived from polyaniline anchored cellulose nanofibers as sustainable microwave absorbers
}

\author{
Avinash R. Pai ${ }^{\mathrm{a}}$, T. Binumol ${ }^{\mathrm{b}}$, Deepu A. Gopakumar ${ }^{\mathrm{c}}$, Daniel Pasquini ${ }^{\mathrm{d}}$, Bastien Seantier ${ }^{\mathrm{e}}$, \\ Nandakumar Kalarikkal ${ }^{\mathrm{a}}$, Sabu Thomas ${ }^{\mathrm{a}, \mathrm{b}, *}$ \\ a International and Inter University Centre for Nanoscience and Nanotechnology, Mahatma Gandhi University, Kottayam, Kerala 686560, India \\ ${ }^{\mathrm{b}}$ School of Chemical Sciences, Mahatma Gandhi University, Kottayam, Kerala 686560, India \\ ${ }^{\mathrm{c}}$ Université de Toulouse, IMT Mines Albi, RAPSODEE CNRS UMR-5302, Campus Jarlard, F-81013, Albi Cedex 09, France \\ ${ }^{\mathrm{d}}$ Chemistry Institute, Federal University of Uberlandia-UFU, Campus Santa Monica-Bloco1D-CP 593, Brazil \\ ${ }^{\mathrm{e}}$ Univ. Bretagne Sud, UMR CNRS 6027, IRDL, F-56100, Lorient, France
}

Keywords:

Cellulose nanofibers

Sustainable aerogels

Polyaniline

Electromagnetic interference shielding

Microwave absorption

Heat dissipation

\begin{abstract}
A B S T R A C T
Electromagnetic (EM) pollution is ubiquitous and has soared to a great extent in the past few decades. The use of plant sourced cellulose nanofibers to fabricate sustainable and high performance electromagnetic shielding materials is foreseen as a green solution by the electronics industry to address this unseen pollutant. In this view, we report a facile and environmentally benign strategy to synthesize ultra-light and highly conductive aerogels derived from cellulose nanofibers (CNF) decorated with polyaniline (PANI) via a simple in-situ polymerization and subsequent freeze drying process devoid of any volatile organic solvents. The obtained conductive aerogels exhibited density as low as $0.01925 \mathrm{~g} / \mathrm{cc}$ with a maximum EMI shielding value $-32 \mathrm{~dB}$ in $\mathrm{X}$ band region. These porous shields demonstrated strong microwave absorption behavior (95\%) with minimal reflection (5\%) coupled with high specific EMI SE value $\sim 1667 \mathrm{~dB} . \mathrm{cm}^{3} \cdot \mathrm{g}^{-1}$ which make these aerogels a potential candidate for use in telecommunication, military and defense applications.
\end{abstract}

\section{Introduction}

The modern electronic era has propelled a rapid upsurge of embedding nano electronic modules into lesser confined spaces for designing advanced functional electronic gadgets. This has increased the chances for intermixing of electromagnetic signals emitted out from these electronic modules thereby deteriorating the performance and life span of the electronic devices. Thus, Electro Magnetic Interference (EMI) shielding is a much sought out solution for the smooth functioning of devices operating in the telecommunication, military and defense sectors. The excess electromagnetic energy emanated from electronic modules is also believed to cause adverse effect on human health (Erogul et al., 2006; Zamanian \& Hardiman, 2005). In the past few decades, thin metal sheets were used to screen EM radiations but are now obsolete as they possess many inherent drawbacks such as poor processability, poor corrosion resistance and higher densities. Conducting polymer nanocomposites containing carbonaceous nanofillers such as CNTs and graphene have received burgeoning research interests to replace conventional metallic shields due to their high end mechanical and electrical properties (Al-Saleh \& Sundararaj, 2009; Chen et al., 2016; Shen, Zhai, \& Zheng, 2014; Yan et al., 2015; Yang, Gupta, Dudley, \& Lawrence, 2005; Zeng et al., 2016). Moreover, intrinsically conducting polymers such as Polypyrrole (PPy) (Guo et al., 2017; Kaur, Ishpal, \& Dhawan, 2012), Polythiophenes (PTs) (Dar et al., 2017; Kulkarni et al., 2019) Poly(3,4-ethylenedioxythiophene) (PEDOT) (Dalal et al., 2019; Luo, Zhang, Mei, Chang, \& Yan, 2016) and Polyaniline (PANI) (Joseph, Varghese, \& Sebastian, 2015; Zhang et al., 2019) has also been of great interest for EMI shielding applications. Very recently, 2D transition metal carbides and nitrides (MXene) have gathered much attention for developing next generation high performance EMI shielding materials (Cao et al., 2018; Sun et al., 2017; Wang et al., 2019; Weng et al., 2018; Zhao et al., 2018). The formation of a well-defined percolating network of these nano inclusions within polymer matrices is of paramount importance to attenuate the incoming electromagnetic radiations (Zeng et al., 2016). In addition, creation of conducting porous architectures is yet another strategy to reduce the density and fabricate lightweight EM shields by tuning the impedance mismatch thereby minimizing the reflection of incident EM

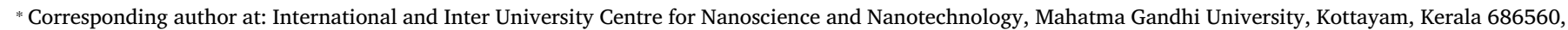
India.

E-mail address: sabuthomas@mgu.ac.in (S. Thomas). 
radiations (Wu, Xie, Sun, Wang, \& Wang, 2015). This is also highly desirous for the present day electronic devices as it lessens the chances of further electromagnetic pollution. Among the family of ICPs, PANI has been extensively studied owing to their tunable electrical conductivity, facile and low cost synthesis with high degree of environmental stability. It has found wide range of applications as sensors (Khadieva et al., 2019; Park et al., 2015), supercapacitors (Eftekhari, Li, \& Yang, 2017; Li, Gao, Wang, Zhang, \& Ma, 2016), elector-chromic devices (Tian et al., 2017; Wu et al., 2016), light emitting diodes (Mohsennia, Bidgoli, Boroumand, \& Nia, 2015; Zhu et al., 2018) and as EMI shields (Gopakumar et al., 2018; Mondal et al., 2017). However, PANI has inherent drawbacks such as poor processability and poor mechanical properties which restricts its use in many high end electronic applications. In order to tackle this issue, numerous works has been reported in the past to develop robust PANI composites with rubbers, thermoplastics and nanocellulose which offer superior processability and durability. Amongst them, cellulose nanofibers have gained much attention due to their inherent properties such as biodegradability, high tensile modulus, high aspect ratio and surface area. This makes nanocellulose as an effective and worthy substrate for PANI to fabricate processable and robust composites.

On the other hand cellulose nanofiber aerogels are a class of hierarchical porous constructs wherein a three dimensional porous architecture is derived by replacing the liquid phase from gels with air via a freeze drying or super critical drying process (Kobayashi, Saito, \& Isogai, 2014; Zhou, Zhang, Lu, Lan, \& Yuan, 2014). These highly porous solids possess many unique characteristics viz. large open pores with ultra-low density and high specific surface area. However, incorporating conducting nano inclusions into aerogels is considered as one of the most impending strategies to significantly reduce the density of new generation electromagnetic shields. Only limited studies have been reported on the electromagnetic shielding efficacy of cellulose based aerogels. Huang et al. first reported the usage of cellulose-CNT aerogels as efficient electromagnetic interference shielding material. The aerogels with density as low as $0.095 \mathrm{~g} . \mathrm{cm}^{-1}$ exhibited an EMI shielding effectiveness of ca. $-20 \mathrm{~dB}$ with a specific EMI shielding effectiveness as high as $219 \mathrm{~dB} \cdot \mathrm{cm}^{3} \cdot \mathrm{g}^{-1}$ (Huang et al., 2015). Graphene oxide-cellulose based hybrid aerogels were also studied to discern its EMI shielding performance in the $\mathrm{X}$ band region. These aerogels exhibited high electrical conductivity value of $19.1 \mathrm{~S} / \mathrm{m}$ with a maximum shielding efficacy value of $58.4 \mathrm{~dB}$ (Wan \& Li, 2016). In this context, cellulose nanofiber-PANI aerogels can be considered as a class of porous constructs that can offer a green and environmentally benign solution to fabricate conductive porous networks devoid of any carbonaceous nano fillers. This was our impetus to develop a sustainable microwave absorbing aerogel for effective suppression of EM waves with an absorption dominant nature in the $\mathrm{X}$ band region.

Initial studies from our group by Gopakumar et al., reported flexible PANI/CNF nanopapers with a total shielding effectiveness value of ca. $-23 \mathrm{~dB} \& 87 \%$ microwave absorption in the $\mathrm{X}$ band region (Gopakumar et al., 2018). Porosity of a material has a pivotal role in enhancing the overall shielding and microwave absorption performance of EM shields (Wu et al., 2015). Henceforth in the present work, we report an array of highly porous and conductive cellulose nanofiberpolyaniline aerogels for potential use as sustainable microwave absorbers. These aerogels demonstrated an ultra-low density of $0.01925 \mathrm{~g} . \mathrm{cm}^{-3}$ with a total shielding efficacy value of ca. $-32 \mathrm{~dB} \& 95$ $\%$ microwave absorption in the $\mathrm{X}$ band region. However, to the best of our knowledge \& literature survey, this is so far the first report on PANI anchored CNF aerogel with superior microwave absorption \& shielding performance in the $\mathrm{X}$ band $(8.2-12.4 \mathrm{GHz})$ region. The prime novelty of this study when compared to our previous report is that PANI/CNF aerogels showcased 1.39 times higher shielding value and superior microwave absorption than PANI/CNF nanopapers at 1:1 ratio. It is also noteworthy that the demonstrated PANI/CNF aerogels exhibited a high specific EMI shielding effectiveness value of $\sim 1667 \mathrm{~dB} \cdot \mathrm{cm}^{3} \cdot \mathrm{g}^{-1}$ which is also one of the highest value reported for non-carbonized CNF aerogels. Moreover, this is also the first report which demonstrates an ultra-fast heat dissipation behavior and real time shielding performance of PANI/CNF aerogels using a mobile phone which portrays the tremendous potential of these porous shields in a real life scenario.

\section{Experimental}

\subsection{Materials and methods}

Ammonium persulfate ( $\geq 98 \%$ purity), Aniline ( $\geq 99.5 \%$ purity), and Hydrochloric acid (35\%) were procured from Sigma Aldrich, India. Cellulose Nanofibers with an average diameter of $20-30 \mathrm{~nm}$ was obtained from eucalyptus bleached Kraft pulp and was supplied by SUZANO, Brazil. CNF was produced by mechanical grinding of the Bleached Eucalyptus Kraft pulp (BEKP) with almost 85-90 \% cellulose, $10-15 \%$ hemicellulose \& $0 \%$ Lignin content (Kappa Number $<1$ ). The Zeta Potential of CNF suspension was around $-36.1 \mathrm{mV}$ and $\sim 0.048 \mathrm{mM} / \mathrm{g}$ of $\mathrm{COOH}$.

\subsection{Fabrication of PANI anchored CNF suspension and Aerogel}

In this study, oxidative polymerization of aniline monomer was initiated onto the surface of cellulose nanofibers via an in-situ technique. Herein, a $20 \mathrm{~g}$ CNF suspension $(3.0 \mathrm{~g} / \mathrm{mL})$ was used for fabrication of thick aerogels with good dimensional stability. Initially, calculated quantities of aniline was mixed with $1 \mathrm{M} \mathrm{HCl}$ solution and cooled down below $5{ }^{\circ} \mathrm{C}$ in an ice bath under constant stirring. This solution was then slowly added to CNF suspension $(0.03 \mathrm{~g} / \mathrm{mL})$ and homogenized at $3000 \mathrm{rpm}$ using an ultra turrax homogenizer to ensure uniform dispersion of aniline in CNF suspension. The oxidant solution was then prepared by dissolving $0.2 \mathrm{M}$ APS in $1 \mathrm{M} \mathrm{HCl}$. This was followed by slow drop wise addition of oxidant solution to the aniline coated CNF suspension kept below $5{ }^{\circ} \mathrm{C}$ in an ice bath with continuous stirring to initiate the polymerization. In all the reaction scheme, APS:aniline ratio was 1:1 and kept constant. After $6 \mathrm{~h}$, a dark green colored PANI/CNF suspension was obtained which was then rinsed with deionised water, acetone and $0.1 \mathrm{M} \mathrm{HCl}$ to remove the unreacted aniline monomer. The washed PANI/CNF suspension was then cooled at $-80^{\circ} \mathrm{C}$ for $4 \mathrm{~h}$ and subsequently lyophilized at $-85^{\circ} \mathrm{C}$ at $0.05 \mathrm{mbar}$ for $48 \mathrm{~h}$ to form robust PANI/CNF aerogels.

\subsection{Characterization \& measurements of the fabricated PANI/CNF aerogels}

The porous architecture of the fabricated PANI/CNF aerogels were investigated by probing the cross-sections of the aerogels under a Field Emission-Scanning Electron Microscopy coupled with EDS (CARL ZEISS, EVO MA10) with an accelerating voltage of $15 \mathrm{kV}$. Prior to FESEM analysis, the aerogel samples were cryo-fractured in liquid nitrogen and sputtered with gold in argon atmosphere. The surface composition analysis of these aerogels were performed using XPS (Model: Axis Ultra DLD, Kratos Analyticals, UK) within the binding energy ranging from 0 to $1000 \mathrm{eV}$. The DC conductivity studies of $\mathrm{PANI} / \mathrm{CNF}$ aerogels was performed by four probe technique using a Keitheley 2400 source meter coupled with a gold probe. The electrical conductivity measurements were performed at room temperature under ambient conditions. The FTIR Spectra of neat CNF aerogel, PANI powder and PANI/CNF aerogels were recorded in transmission mode using a Fourier transform infrared spectro photometer, IR prestige-21, shimadzu in the range $400-4000 \mathrm{~cm}^{-1}$ with a resolution of $2 \mathrm{~cm}^{-1}$ at 15 scans for each sample. The $\mathrm{x}$ ray diffractograms of neat CNF aerogel, PANI powder and PANI/CNF aerogels were obtained with SHIMADZU XRD-6000. The study was conducted at ambient conditions within in a $2 \Theta$ range from 5 to $40^{\circ}$ at a scan rate of $2^{\circ} \mathrm{min}^{-1}$. The electromagnetic interference shielding performance of the fabricated PANI/CNF 
aerogels was measured using a two port PNA Network analyzer (model: Agilent E8362B, Freq: $10 \mathrm{MHz}-20 \mathrm{GHz}$ ) which was coupled to a Keycom waveguide functioning in $\mathrm{X}$ band region $(8.2-12.4 \mathrm{GHz})$. The scattering parameters of the samples were traced and the total shielding effectiveness of the PANI/CNF aerogels were calculated using standard equations. The thermal images of the fabricated aerogels were taken using an IR camera (FLIR E5). The samples were placed inside a microwave oven and irradiated for $6 \mathrm{~s}$ and were taken out for thermal imaging. The thermal images were taken in an interval of $5 \mathrm{~s}$. The Brunauer-Emmett-Teller (BET) surface area of the fabricated PANI/ $\mathrm{CNF}$ aerogels were determined by $\mathrm{N}_{2}$ physisorption using a Belsorp-Max automated system. Nearly $0.1-0.2 \mathrm{~g}$ of aerogel sample was initially degassed in the sample chamber at $120^{\circ} \mathrm{C}$ for $4 \mathrm{~h}$ prior to the analysis followed by $\mathrm{N}_{2}$ adsorption at $-196^{\circ} \mathrm{C}$. The thermogravimetric analysis of PANI/CNF aerogels were performed using SDT Q600 analyzer (TA Instruments) in the range of $30-700{ }^{\circ} \mathrm{C}$ with a heating rate of $10{ }^{\circ} \mathrm{C} /$ min. The density of PANI/CNF aerogels $(\rho)$ were determined by measuring the volume and weight of the aerogel sample with a dimension $10 \mathrm{~mm} * 10 \mathrm{~mm} * 5 \mathrm{~mm}$ by using the equation $\left(\rho=\frac{M_{0}}{V}\right)$ where, $M_{0}$ is the weight and V is the volume of PANI/CNF aerogel. Prior to the estimation of density, the PANI/CNF samples were dried in an oven at $60^{\circ} \mathrm{C}$ for $6 \mathrm{~h}$ to remove the residual moisture in the samples. The average density of five samples were determined and reported. The porosity of PANI/CNF aerogels were calculated by using the equation $\left(1-\frac{\rho}{\rho_{T}}\right)$ * $100 \%$, where $\rho$ is the average density of PANI/CNF aerogel and $\rho_{T}$ is the theoretical density of nanocellulose.

\section{Results and discussion}

A series of conductive, lightweight PANI/CNF aerogels were fabricated as per the strategy depicted in Fig. 1. Polymerization of aniline monomer was initiated in-situ by drop wise addition of APS oxidant to subsequently for a uniform coating of polyaniline onto the surface of the cellulose nanofibers as shown in Fig. 2 (a). The resultant PANI/CNF suspensions were then washed with acetone, $0.1 \mathrm{M} \mathrm{HCl} \&$ deionised water to remove untreated monomer from the suspension. The resultant $\mathrm{PANI} / \mathrm{CNF}$ suspension was then freeze dried at $-80{ }^{\circ} \mathrm{C}$ at $0.03 \mathrm{mbar}$ for $48 \mathrm{~h}$ to form a series of conducting PANI/CNF aerogels. Fig. 2(b), represents the possible ways of secondary interactions with PANI and CNF which plays a crucial role in the formation of a continuous conductive web like structures within the PANI/CNF aerogels.

\subsection{FTIR analysis}

Initially, FTIR studies have been done to elucidate the structure of PANI, CNF and PANI/CNF aerogels and the secondary interactions within PANI and CNF. As shown in Fig. 3(a), pristine CNF shows characteristic bands at 3340, 2895, 1629, 1428, 1321, 1161, 1025 \& $899 \mathrm{~cm}^{-1}$. The peak at $3340 \mathrm{~cm}^{-1}$ can be attributed to $\mathrm{O}-\mathrm{H}$ stretching. The bands at $2895 \& 1629 \mathrm{~cm}^{-1}$ arises due to the $\mathrm{C}-\mathrm{H}$ stretching and $\mathrm{H}-\mathrm{O}-\mathrm{H}$ bending of the water molecules adsorbed inside CNF. The peaks at $1321 \& 1161 \mathrm{~cm}^{-1}$ corresponds to $\mathrm{O}-\mathrm{H}$ bending and $\mathrm{C}-\mathrm{O}$ antisymmetric bridge stretching. A strong band around $1025 \mathrm{~cm}^{-1}$ arises due to $\mathrm{C}-\mathrm{O}-\mathrm{C}$ pyranose ring skeletal vibration $(\mathrm{Wu}, \mathrm{Lu}, \mathrm{Xu}$, Zhang, \& Zhou, 2014). Pristine PANI also shows its characteristic peaks at $3328,1599,1489,1298,1160 \& 788 \mathrm{~cm}^{-1}$. The peak at $3328 \& 1160$ $\mathrm{cm}^{-1}$ can be ascribed to the $\mathrm{N}-\mathrm{H}$ stretching. The peaks at $1298 \& 1160$ $\mathrm{cm}^{-1}$ can be assigned to $\mathrm{C}-\mathrm{N}$ stretching and $\mathrm{C}-\mathrm{H}$ vibration in the benzene ring. The peaks at $1599 \& 1489 \mathrm{~cm}^{-1}$ can be attributed to the stretching vibration of benzenoid and quinoid moities in the PANI ( $\mathrm{Hu}$, Chen, Yang, Liu, \& Wang, 2011). The presence of benzenoid and quinoid moieties also confirm that the PANI is in its emeraldine form. The FTIR spectrum of PANI/CNF aerogels appears to be a superposition of the FTIR spectra of pristine CNF and PANI. It is noteworthy that, in case of 0.2:1 \& 0.5:1 PANI/CNF aerogels, the peak intensities of $\mathrm{O}-\mathrm{H}$ stretching $\left(3340 \mathrm{~cm}^{-1}\right)$ in $\mathrm{CNF}$ and $\mathrm{N}-\mathrm{H}\left(3328 \mathrm{~cm}^{-1}\right)$ stretching in PANI decreases drastically that can be ascribed due to the strong intermolecular forces of interaction between PANI and CNF (Hu et al.,

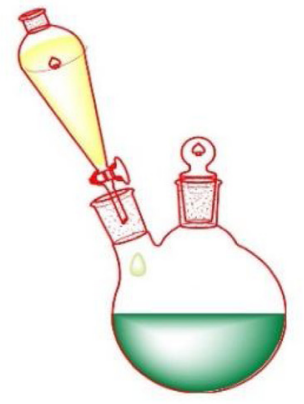

Addition of aniline to CNF suspension

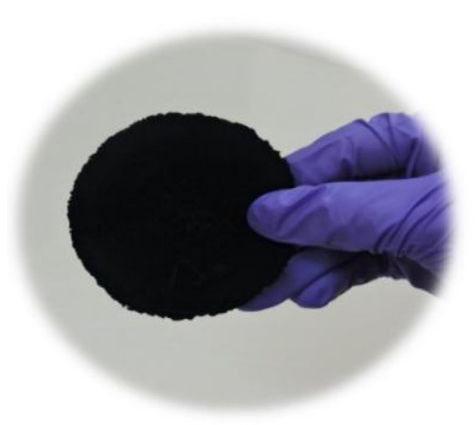

PANI/CNF Aerogel

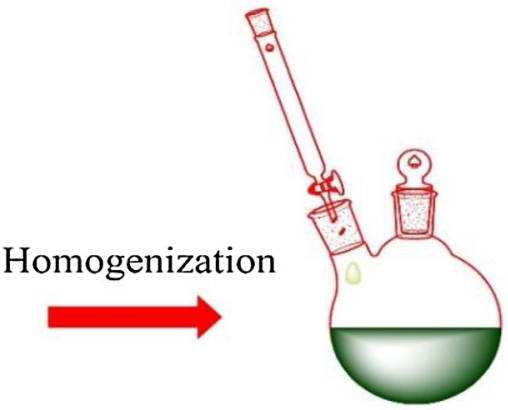

Addition of APS initiator

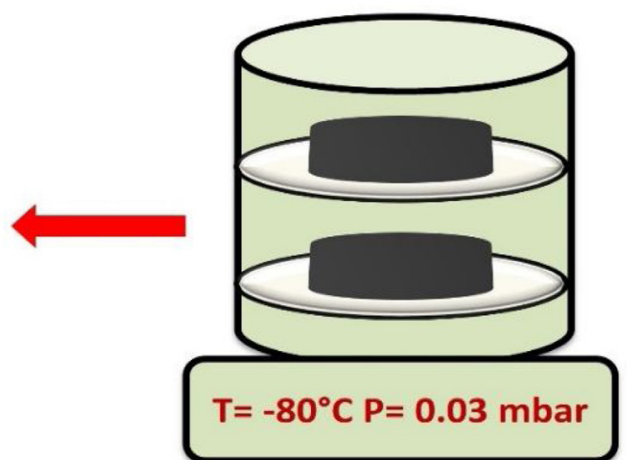

Freeze Drying (48 Hrs)
In-situ polymerization

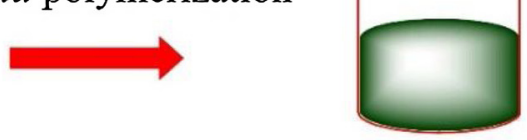

PANI/CNF Suspension

Fig. 1. A schematic depicting the steps involved in the fabrication of PANI/CNF aerogels. 

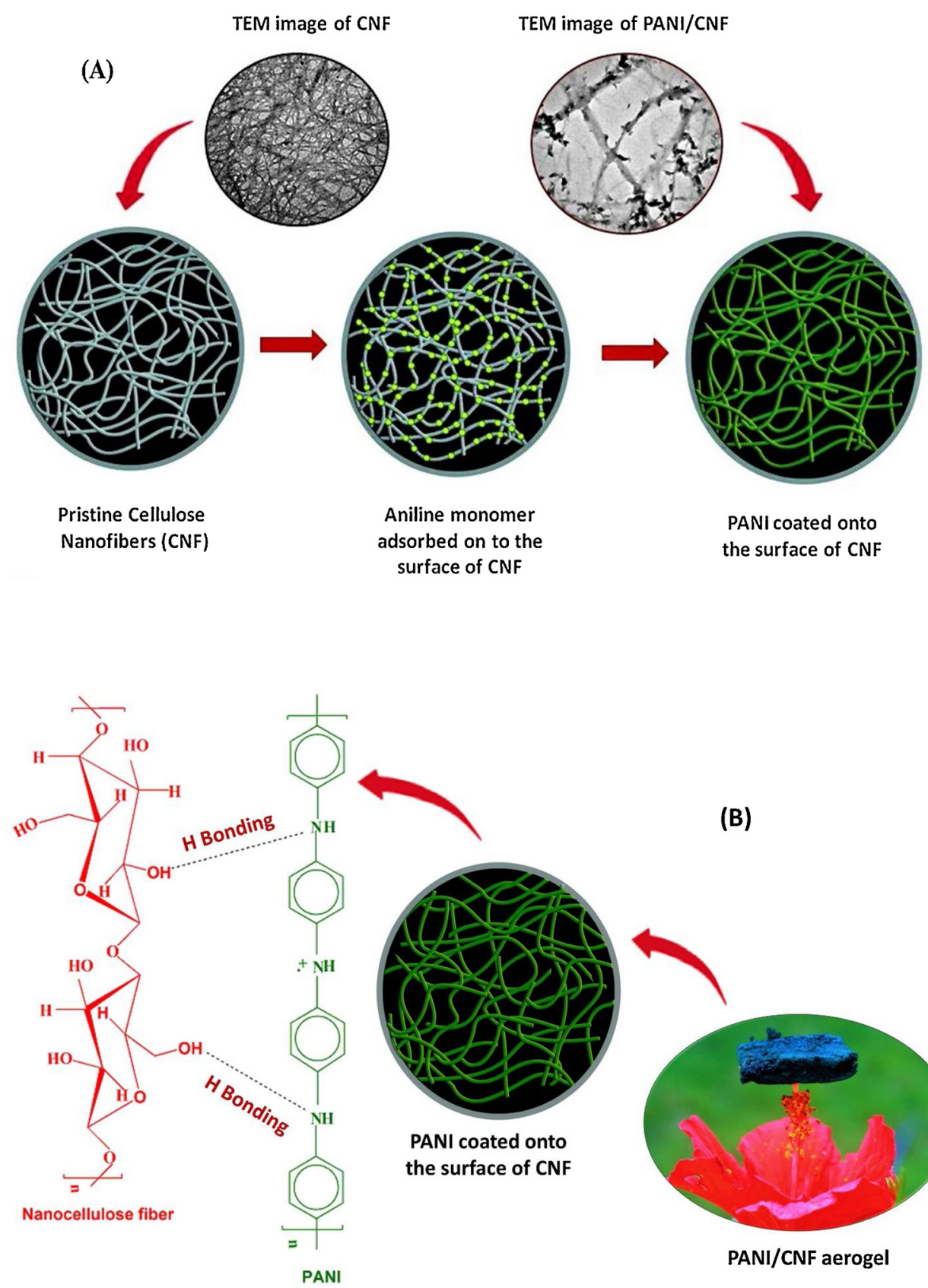

(B)

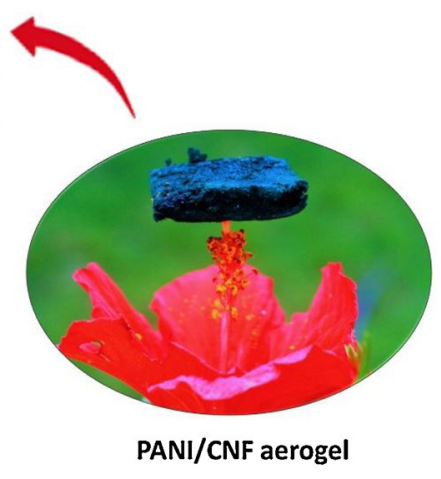

Fig. 2. (a) The reaction scheme for in-situ polymerization of aniline monomer onto the surface of cellulose nanofibers. (b) Specific secondary interactions between PANI and CNF and a photograph depicting lightweight nature of PANI/CNF aerogel.

2011). It can also be seen that for $1: 1$ PANI/CNF aerogels, the characteristic peaks of CNF (3340 \& $1025 \mathrm{~cm}^{-1}$ ) and PANI (1599, 1489 \& $1160 \mathrm{~cm}^{-1}$ ) was shifted to lower wavenumbers (red shift) in PANI/CNF aerogel $\left(3281,1576,1481,1150 \& 1019 \mathrm{~cm}^{-1}\right.$ ) which confirms the existence of strong intermolecular hydrogen bonding between CNF and PANI in PANI/CNF aerogels (Luo et al., 2018; Wu et al., 2014; Zhou et al., 2014)

\subsection{XPS analysis}

The XPS analysis of PANI/CNF aerogels were performed to elucidate the type and nature of chemical bonds formed after in-situ polymerization of aniline monomer on the CNF surface. The wide scan survey spectra of 1:1 (PANI:CNF) aerogel clearly shows the presence of elements like $\mathrm{C}, \mathrm{O}$ and $\mathrm{N}$ which proves the formation of poly(aniline) in the fabricated aerogels. As seen from Fig. 4(a) the peaks at $284.9 \mathrm{eV}$,
$398.9 \mathrm{eV} \& 531.9 \mathrm{eV}$ corresponds to C1s, N1s and O1s respectively (Shi et al., 2012). The C1s spectrum of PANI/CNF aerogel shows four distinct peaks at $284.6 \mathrm{eV}(\mathrm{C}-\mathrm{C}), 286.1 \mathrm{eV}(\mathrm{C}-\mathrm{N}) .287 .4 \mathrm{eV}(\mathrm{C}=\mathrm{O})$ \& $288.6 \mathrm{eV}(\mathrm{O}-\mathrm{C}-\mathrm{O})$. The presence of $\mathrm{C}-\mathrm{N}$ bond also indicates the presence of intermolecular hydrogen bonding between the $-\mathrm{OH}$ groups of $\mathrm{CNF}$ and $\mathrm{N}-\mathrm{H}$ group of PANI and are in good agreement with the findings from FTIR analysis (Luo et al., 2018; Shi et al., 2012). The N1s spectra has three peaks centered at $398.8 \mathrm{eV}$ (Quinoid imine, $=\mathrm{N}-$ ), $399.8 \mathrm{eV}$ (Benzoid amine, $-\mathrm{NH}-$ ) \& $401.8 \mathrm{eV}$ (Positively charged Nitrogen, $\mathrm{N}^{+}$) which also confirms the formation of protonated emeraldine salt form of PANI which imparts good electrical conductivity to the fabricated PANI/CNF aerogels. The O1s spectrum also shows three peaks at $530.5 \mathrm{eV}(\mathrm{C}=\mathrm{O}), 532.3(\mathrm{C}-\mathrm{OH}$ and/or $\mathrm{C}-\mathrm{O}-\mathrm{C}) \& 533.8 \mathrm{eV}$ which corresponds to water molecules or chemisorbed oxygen in the PANI/CNF aerogel. 

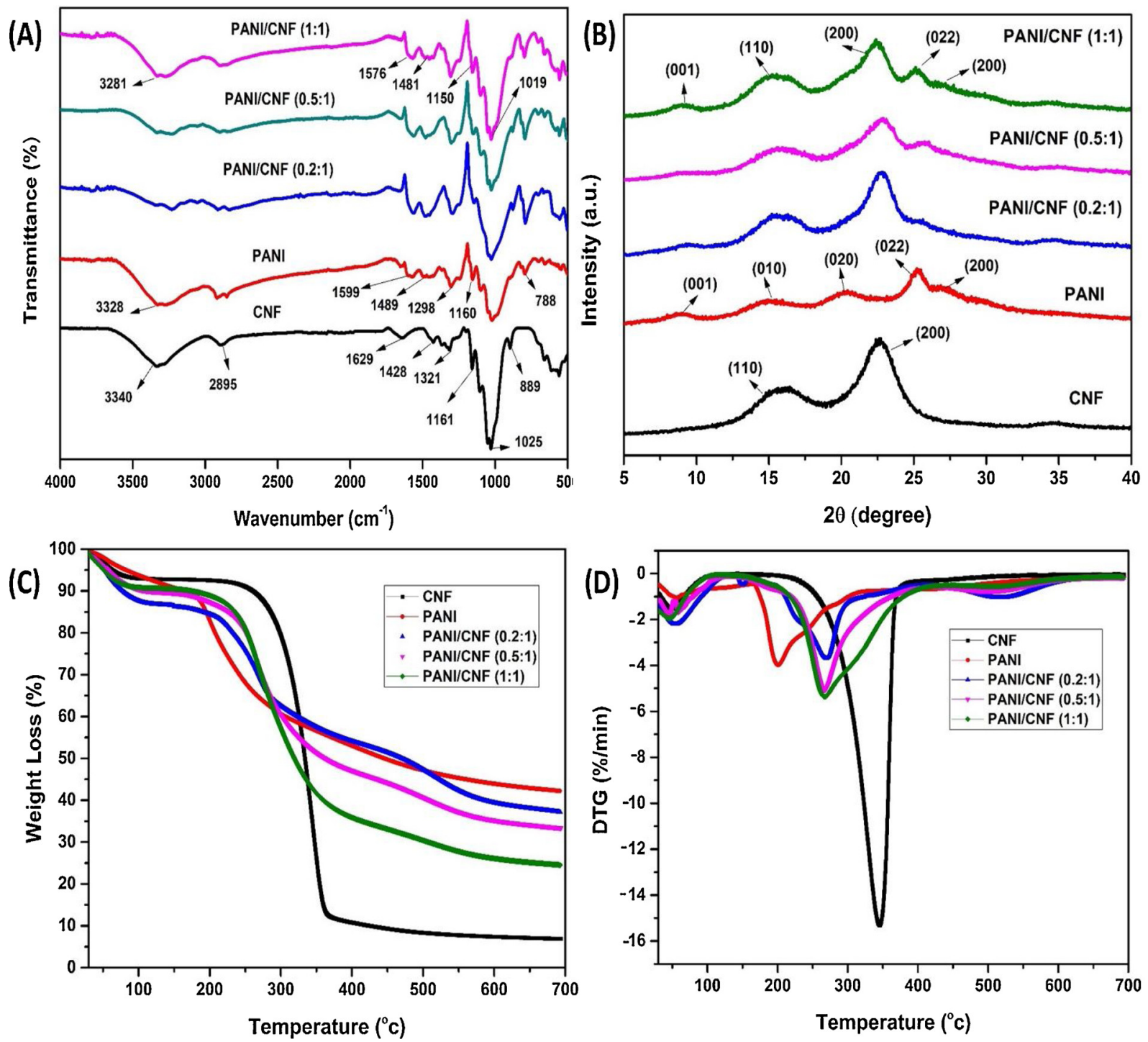

Fig. 3. (a) FTIR Spectra (b) XRD Spectra (c) TGA traces \& (d) DTG curves of CNF aerogel, PANI powder, (0.2:1), (0.5:1) \& (1:1) PANI/CNF aerogels.

\subsection{XRD analysis}

The XRD patterns of pristine PANI powder, CNF aerogel and PANI/ CNF composite aerogel is shown in the Fig. 3(b). In the case of the XRD patterns of neat CNF, it displayed two main peaks at 16.3 and $22.6^{\circ}$ have Miller indices of (110) and (200), respectively indicates the typical cellulose-I structure which implies that the in-situ polymerization of aniline monomer did not affect the structure of the cellulose nanofiber (French, 2014; Gopakumar et al., 2018). In the case of PANI powder, it exhibited five crystalline peaks at $2 \theta 9.73^{\circ}, 15.1^{\circ}, 20.4^{\circ}, 25.2^{\circ}$ and $26.81^{\circ}$ with a broad background corresponding to (001), (010), (020), (022) and (200) reflections of pristine polyaniline in its emeraldine salt form (Yu, Chen, Chen, \& Liu, 2014). The characteristic peaks at $9.73^{\circ}$, $15.1^{\circ}$ and $20.4^{\circ}$ were attributed to the periodic channels parallel to the principal chain. The peak at $25.2^{\circ}$ and $26.81^{\circ}$ were due to the periodic arrays perpendicular to the principal chain. As the concentration of PANI increases in PANI/CNF aerogels, the intensity of the peak at $25.2^{\circ}$ (022) also increases which signifies that the crystal structure of both PANI and CNF coexist in the composite aerogels. This also implies that the XRD spectra of PANI/CNF aerogel exhibits a superimposed spectrum of PANI and CNF diffraction profiles.

\subsection{TGA analysis}

Thermogravimetric analysis was performed to investigate the thermal stability \& degradation behavior of pristine CNF, PANI and PANI/CNF aerogels. Fig. 3(c) and (d) illustrates the TGA and DTG curves of pristine CNF, PANI and PANI/CNF aerogels. In the case of all the samples there was slight weight loss that was observed around $100{ }^{\circ} \mathrm{C}$ which can be attributed to the escape of entrapped moisture. For neat CNF, a sharp weight loss can be seen around $240-350{ }^{\circ} \mathrm{C}$ in the DTG curve which can be ascribed to the degradation of crystalline regions of CNF. Neat PANI exhibits a three step degradation process. First around $100^{\circ} \mathrm{C}$ which corresponds to the escape of residual moisture. The second degradation peak around $180-240^{\circ} \mathrm{C}$ which was due to the decomposition of $\mathrm{HCl}$ dopant in PANI. The third peak was in the range of $420-580^{\circ} \mathrm{C}$ that can be assigned for the decomposition of PANI chain backbone (Zhou et al., 2014) Furthermore, the degradation rate of PANI was slower than CNF which showcase good thermal stability of pristine PANI. In the case of 0.2:1, 0.5:1 \& 1:1 PANI:CNF aerogels, the degradation behavior was a similar three step process as observed in PANI. The onset thermo-oxidative degradation temperature of all PANI/CNF aerogels was found to increase with the increasing concentration of PANI. It is also worth mentioning that with the combination of PANI which is more thermally stable, the degradation rate of 

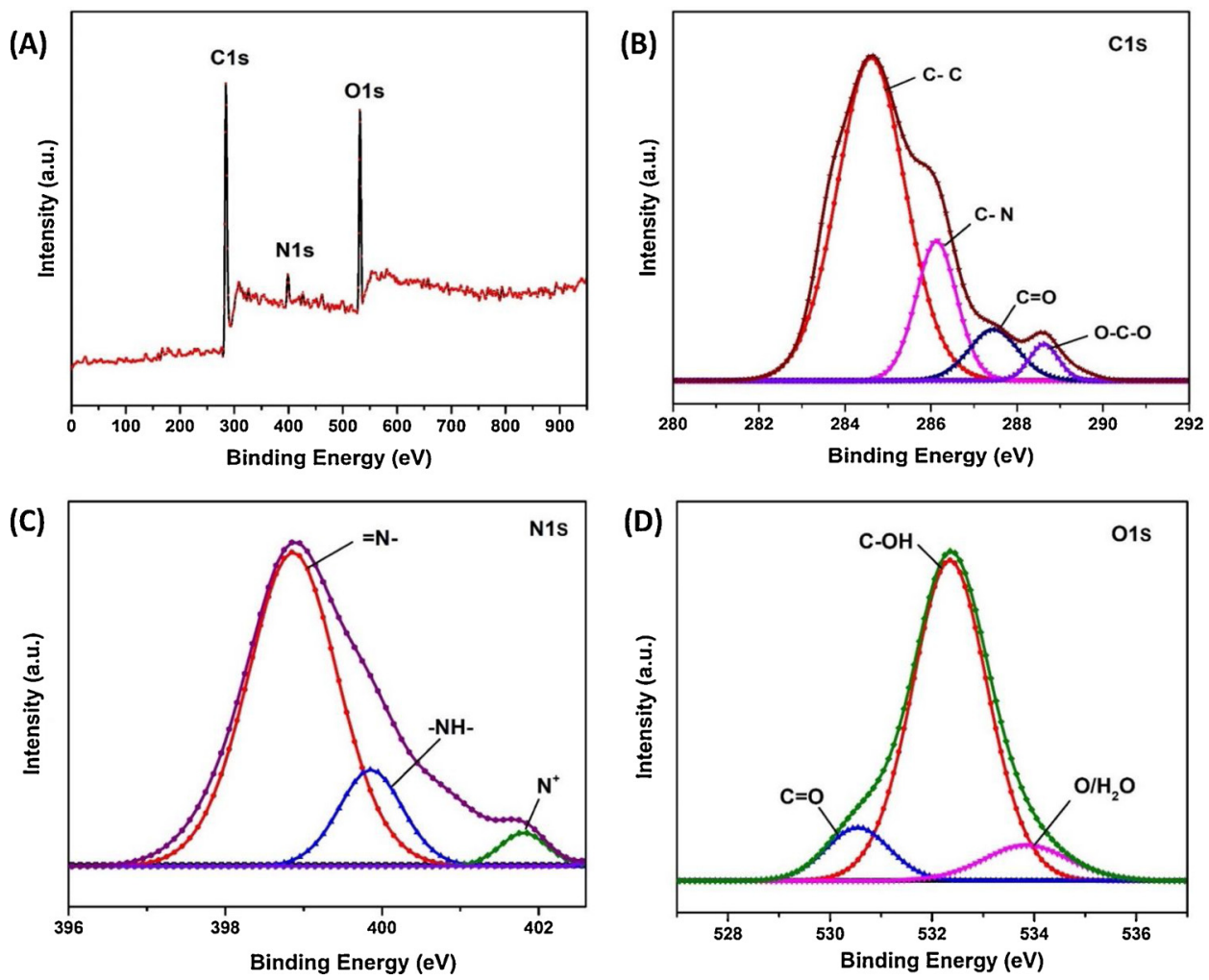

Fig. 4. (a) XPS wide scan spectra (b) C1s spectra (c) N1s spectra \& (d) O1s spectra of 1:1 PANI:CNF aerogel.

PANI/CNF aerogel was found to be slower than the pristine CNF aerogel (Hu et al., 2011). Hence it can be concluded that PANI forms a protective layer onto the CNF and enhances the thermal stability of the fabricated PANI/CNF aerogels.

\subsection{Surface morphology, physical properties \& electrical conductivity of PANI/CNF aerogels}

The surface morphology of all the three compositions viz. 0.2:1, 0.5:1 \& 1:1 PANI:CNF aerogels were observed using FE-SEM. The images were taken at two different magnifications at $30 \mu \mathrm{m}$ and $100 \mathrm{~nm}$ scales to probe into the surface topography of the aerogels. Fig. 5(a), (d) $\&(g)$ clearly depicts the formation of a highly porous 3D interconnected network like structure in PANI/CNF aerogels. After in-situ polymerisation of aniline in CNF suspension, it forms a rough coating of PANI on the surface of CNF. This resulted in the formation of a well interconnected conductive web like structures within PANI/CNF aerogels. These conductive web like structures will now interact with the incoming EM radiations and is of much significance for screening undesired EM signals. Higher resolution images in Fig. 5(b), (e) \& (h) show the presence of PANI anchored on individual CNF surface which also confirms the presence of strong intermolecular forces of interaction between PANI and CNF. Nitrogen elemental mapping of all the fabricated aerogels is shown in Fig. 5(c), (f) \& (i). As the concentration of PANI increases in PANI/CNF aerogels, the green dots corresponding to the nitrogen element (in PANI) also varies in accordance with the monomer content used for the fabrication of PANI/CNF aerogels. To probe deeper into the formation of PANI coating on CNF, TEM images of neat CNF and PANI anchored CNF suspension is given as Fig. 5(j) and (k) (see supporting information). Furthermore, the coating of PANI resulted in a long range $\pi$ conjugation on the surface of cellulose nanofibers that resulted in the excellent electrical conductivity in the resultant aerogels. The density, porosity, BET surface area and DC conductivity values of the fabricated PANI/CNF aerogels are summarized in Table 1. When compared with neat CNF aerogel, PANI/CNF aerogels showcased higher densities that can be ascribed due to the presence of PANI in the aerogels. As the PANI content increases in the PANI/CNF aerogels, the BET surface area was found to increase from $94.8 \mathrm{~m}^{2} / \mathrm{g}$ (of neat CNF) to $109.3 \mathrm{~m}^{2} / \mathrm{g}$. This is due to the fact that PANI forms a rough coating on CNF which in turn enhances the surface area of the PANI/ $\mathrm{CNF}$ aerogels. Similar studies on the enhancement in the specific surface area was reported by Sukun Zhou et al. in silane modified CNF aerogels (Zhou et al., 2016). Moreover, all the fabricated PANI/CNF aerogels exhibited higher porosities in the range of 98.6-99.1\% which is of paramount importance in enhancing the overall shielding performance of PANI/CNF aerogels.

\subsection{Electromagnetic absorption performance of PANI/CNF aerogels}

The microwave supression performance of the fabricated PANI/CNF aerogels were investegated by recording the scattering parameterers and the total shielding effectiveness of the aerogels were expressed in terms of decibels (dB). As per the classical microwave attenuation theories, the condutivity of a material has prime importance in shielding the incoming electomagnetic radiations (Fugetsu et al., 2008). Hence, a long range connectivity of the conducting nanoinclusions within the polymer nanocomposite is very much crucial to fabricate EMI shields with higher shielding effectiveness. In the case of PANI/ $\mathrm{CNF}$ aerogels, the morphological studies revealed that a well defined conductive coating of polyaniline was formed onto the surface of cellulose nanofibers via strong intra molecular hydrogen bonding which makes PANI/CNF aerogels as a potential candidate for supressing electromagentic radiations in $\mathrm{X}$ band region. The EMI sheilding mechanism of any EMI shield is usually the summation of three different mechanisms namely shielding by absorption $\left(\mathrm{SE}_{\mathrm{A}}\right)$, reflection $\left(\mathrm{SE}_{\mathrm{R}}\right)$ and multiple reflections $\left(\mathrm{SE}_{\mathrm{MR}}\right)$. Hence, The total shielding effectiveness ( $\left.\mathrm{SE}_{\mathrm{Total}}\right)$ of PANI/CNF aerogels can be written as

$\mathrm{SE}_{\text {Total }}(\mathrm{dB})=\mathrm{SE}_{\mathrm{A}}+\mathrm{SE}_{\mathrm{R}}+\mathrm{SE}_{\mathrm{MR}}$

In the case of PANI/CNF aerogels, the incoming electomgnetic 

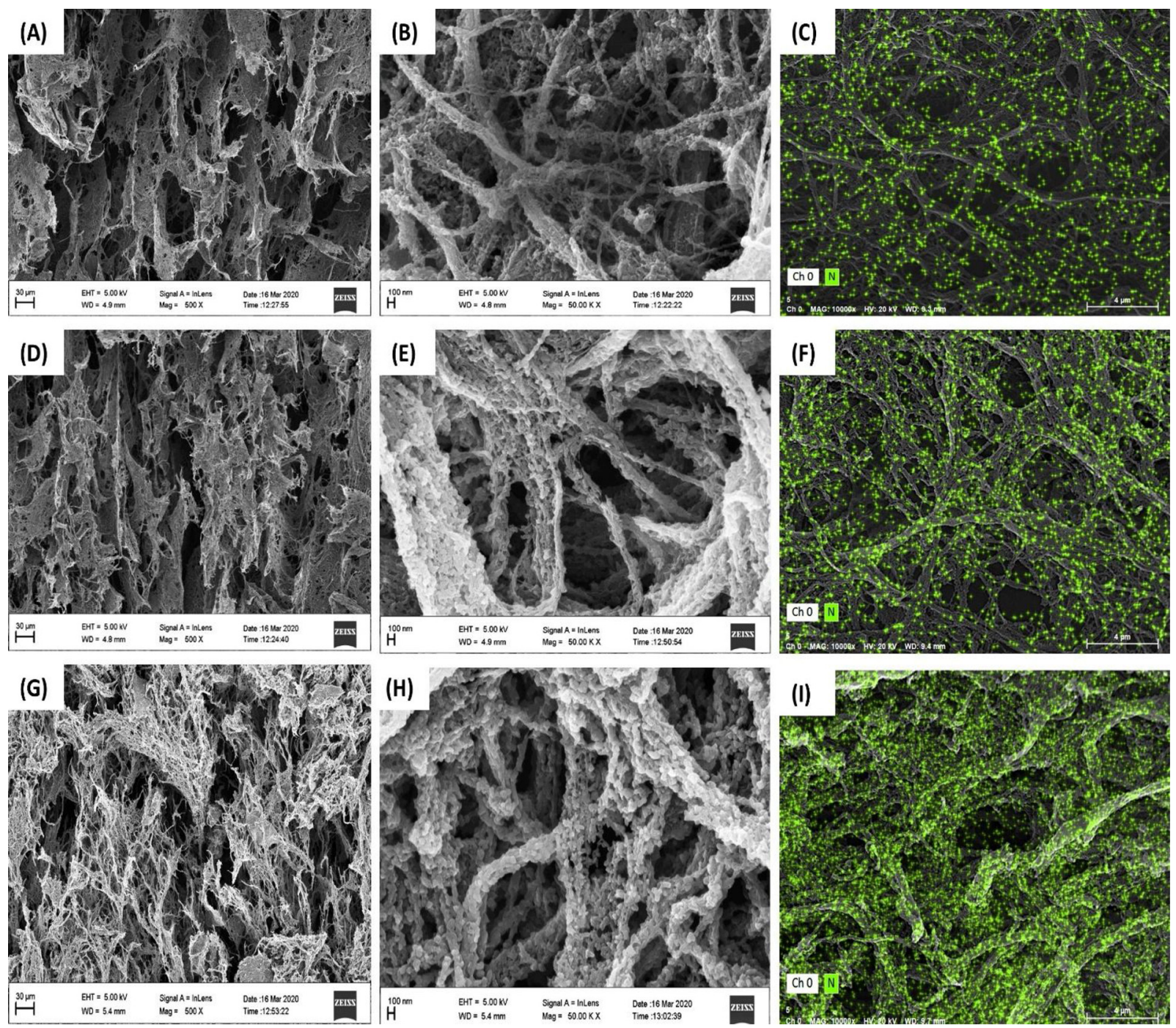

Fig. 5. FE-SEM images at $30 \mu \mathrm{m}$ scale, $100 \mathrm{~nm}$ scale \& EDS Nitrogen Element Mapping of (a)-(c) 0.2:1 (d)-(f) 0.5:1 and (g)-(i) 1:1 PANI:CNF aerogels.

radiations will mostly undergo a mutiple internal refection with in the porous network and the re-reflected waves will be absorbed and dissipated in the form of heat. Hence in this context, the shielding due to multiple internal reflection $\left(S E_{M R}\right)$ component was merged with $S E_{A}$. The total shielding effectiveness of PANI/CNF aerogels can now be reexpressed as Eq. (2)

$\mathrm{SE}_{\text {Total }}(\mathrm{dB})=\mathrm{SE}_{\mathrm{A}}+\mathrm{SE}_{\mathrm{R}}$

The power coefficients of transmittance (T), reflectance (R) and absorbance (A) of a given shielding material can be expressed in terms of scattering parameters as given expressed in Eq. 3-7.

$\mathrm{T}=\left|\mathrm{S}_{12}\right|^{2}=\left|\mathrm{S}_{21}\right|^{2}$

$\mathrm{R}=\left|\mathrm{S}_{11}\right|^{2}=\left|\mathrm{S}_{22}\right|^{2}$

$\mathrm{A}=1-\mathrm{R}-\mathrm{T}$
$S E_{R}=-10 \log (1-R)$

$S E_{A}=-10 \log \left(\frac{T}{1-R}\right)$

Where, $S_{11}, S_{21}, S_{12} \& S_{22}$ are the scattering parameters which were traced from the vector network analyzer (VNA).

The total shielding effectiveness, microwave absorption and reflection components of the fabricated PANI/CNF aerogels in the $\mathrm{X}$ band $(8.2-12.4 \mathrm{GHz})$ region is shown in Fig. 6(a)-(c). The maximum total shielding effectiveness of ca. $-32 \mathrm{~dB}$ was obtained for 1:1 PANI:CNF aerogels at $5.0 \mathrm{~mm}$ thickness @ 12 GHz. The aerogels with 1:0.2, 1:0.5 PANI:CNF aerogels exhibited $\mathrm{SE}_{\mathrm{Total}}$ values of $-13.2 \mathrm{~dB}$ and $-20.8 \mathrm{~dB}$ respectively. The DC conductivity of PANI:CNF aerogels was found to be dependent on the concentration of polyaniline and hence 1:1 (PANI:CNF) aerogels exhibited a maximum value of $0.345 \mathrm{~S} / \mathrm{cm}$ (See

Table 1

Physical \& Electrical properties of the fabricated PANI/CNF aerogels.

\begin{tabular}{|c|c|c|c|c|c|}
\hline Sr No & Aerogel Composition (PANI:CNF) & Density $\left(\mathrm{g} / \mathrm{cm}^{3}\right)$ & Porosity (\%) & BET Surface Area $\left(\mathrm{m}^{2} / \mathrm{g}\right)$ & DC Conductivity (S/cm) \\
\hline 1 & Neat CNF & 0.00712 & 99.5 & 94.8 & $1.0 \times 10^{-13}$ \\
\hline 2 & $0.2: 1$ & 0.01743 & 99.1 & 98.9 & 0.006 \\
\hline 3 & $0.5: 1$ & 0.01892 & 98.9 & 105.7 & 0.091 \\
\hline 4 & $1: 1$ & 0.01925 & 98.6 & 109.3 & 0.345 \\
\hline
\end{tabular}



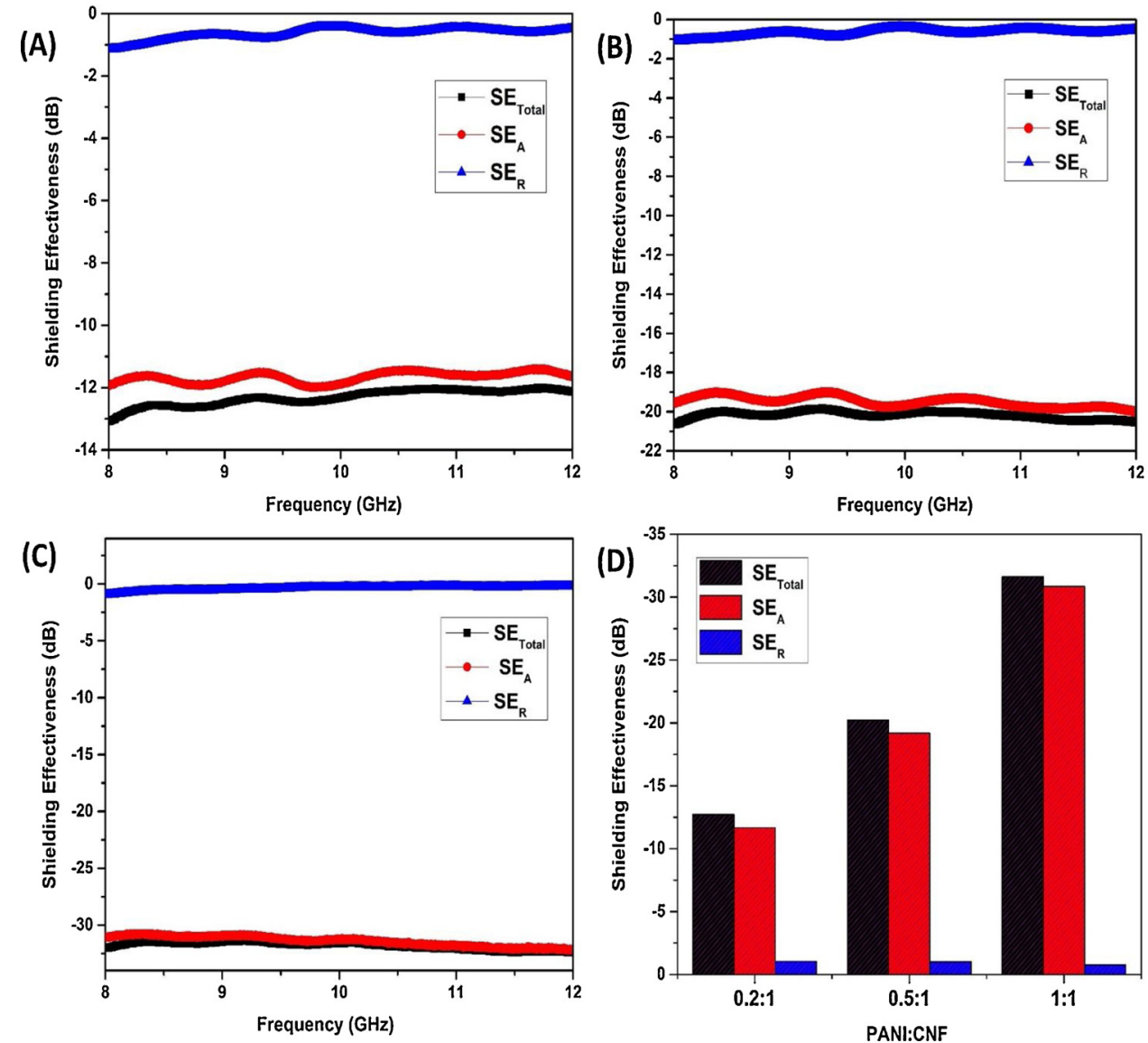

Fig. 6. EMI shielding effectiveness of (a) 0.2:1 (b) 0.5:1 \& (c) 1:1 (PANI:CNF) aerogels with $5.0 \mathrm{~mm}$ thickness in X band region (8.2-12.4 GHz). (d) Electromagnetic Shielding performance $\left(\mathrm{SE}_{\text {total }}, \mathrm{SE}_{\mathrm{A}}, \mathrm{SE}_{\mathrm{R}}\right)$ of the fabricated PANI/CNF aerogels with varying PANI:CNF ratio at $8.2 \mathrm{GHz}$.

Supporting Video S1). The formation of a well-defined porous web like network of polyaniline inside the PANI/CNF aerogels plays a key role in enhancing the electrical conductivity and thereby EMI shielding ability. The inter-molecular hydrogen bonding with the hydroxyl groups of CNF and nitrogen of polyaniline is envisaged for achieving $>99.9 \%$ attenuation for PANI/CNF aerogels. From the Fig. 6(d), it is also worth mentioning that the PANI/CNF aerogels exhibited shielding behavior by a predominant absorption (95\%) mechanism with only minimal reflection $(5 \%)$ of the incoming electromagnetic radiations. Such a mechanism is highly preferred for the development of green and sustainable EMI shielding solutions where it prevents reflecting the misdirected electromagnetic radiations and to stifle electromagnetic pollution. In comparison with our previous study by Gopakumar et al. (2018), the total shielding effectiveness value of PANI/CNF aerogels was found to be 1.39 times higher than the PANI/CNF nanopapers at 1:1 ratio. This can be attributed to the fact that the presence of air pockets at the air/shield interface of PANI/CNF aerogel facilitates better impedance matching and allows the EM waves to enter into the porous network thereby facilitating enhanced EM absorption \& shielding performance. A cartoon depicting the plausible mechanism for shielding by absorption of the incident electromagnetic energy is shown in Fig. 7(a). As the misdirected EM waves enter into the bulk of the aerogel, the electromagnetic waves cannot escape from the pores and undergoes a series of multiple internal scatterings within the 3D porous network and eventually gets absorbed and converted into heat energy due to dipole and interfacial polarization occurring within the PANI/CNF aerogel.

The specific shielding effectiveness (SSE) of the fabricated aerogels was calculated by dividing the total shielding effectiveness value with the density $\left(\mathrm{SSE}=\mathrm{SE}_{\mathrm{Total}} / \rho_{\text {aeogel }}\right)$ of the PANI/CNF aerogels. This value is of great interest for designing lightweight materials for EMI shielding applications. In the present study the fabricated aerogels showcased a high SSE value of $\sim 1667 \mathrm{~dB} . \mathrm{cm}^{3} . \mathrm{g}^{-1}$. in $8.2-12.4 \mathrm{GHz}$ (X Band) region. The ultra-low density $\left(0.01925{\mathrm{~g} . \mathrm{cm}^{-3}}^{-3}\right)$ of the fabricated PANI/ CNF aerogels has led to an increase in the SSE value of the PANI/CNF aerogels. Fig. 7(b), gives a comparative graph indicating the SSE values of various EMI shields reported in the literature with PANI/CNF aerogel. This comparison gives a strong indication that these fabricated aerogels have a tremendous potential as high performance and lightweight green microwave absorbers.

However, there are only limited studies reported on nanocellulose based aerogels as high power microwave absorbers. The key challenge for fabricating such green absorbers from nanocellulose is to fine tune the electrical conductivity of these porous materials. However, in the case of PANI/CNF aerogels the uniform coating of polyaniline has been attributed for the enhanced EMI shielding effectiveness of $-32 \mathrm{~dB} @$ 8.2 GHz. Table 2, manifests some of the previous reported works on cellulose derived bio aerogels and their corresponding attenuation performance, electrical conductivity, density and the frequency region at which the samples were tested. Hence, from the reported values one can conclude that PANI/CNF aerogels are potential candidates for EMI shielding applications. In addition, attaining an effective EMI SE value of $>20 \mathrm{~dB}$ is considered as worthy for its use in electronic gadgets for commercial applications.

\subsection{Skin effect of PANI/CNF aerogel}

Shield thickness is one of the crucial factors which decides the overall attenuation performance of any EMI shielding material. Hence there exists a critical thickness at which the intensity of the incident 


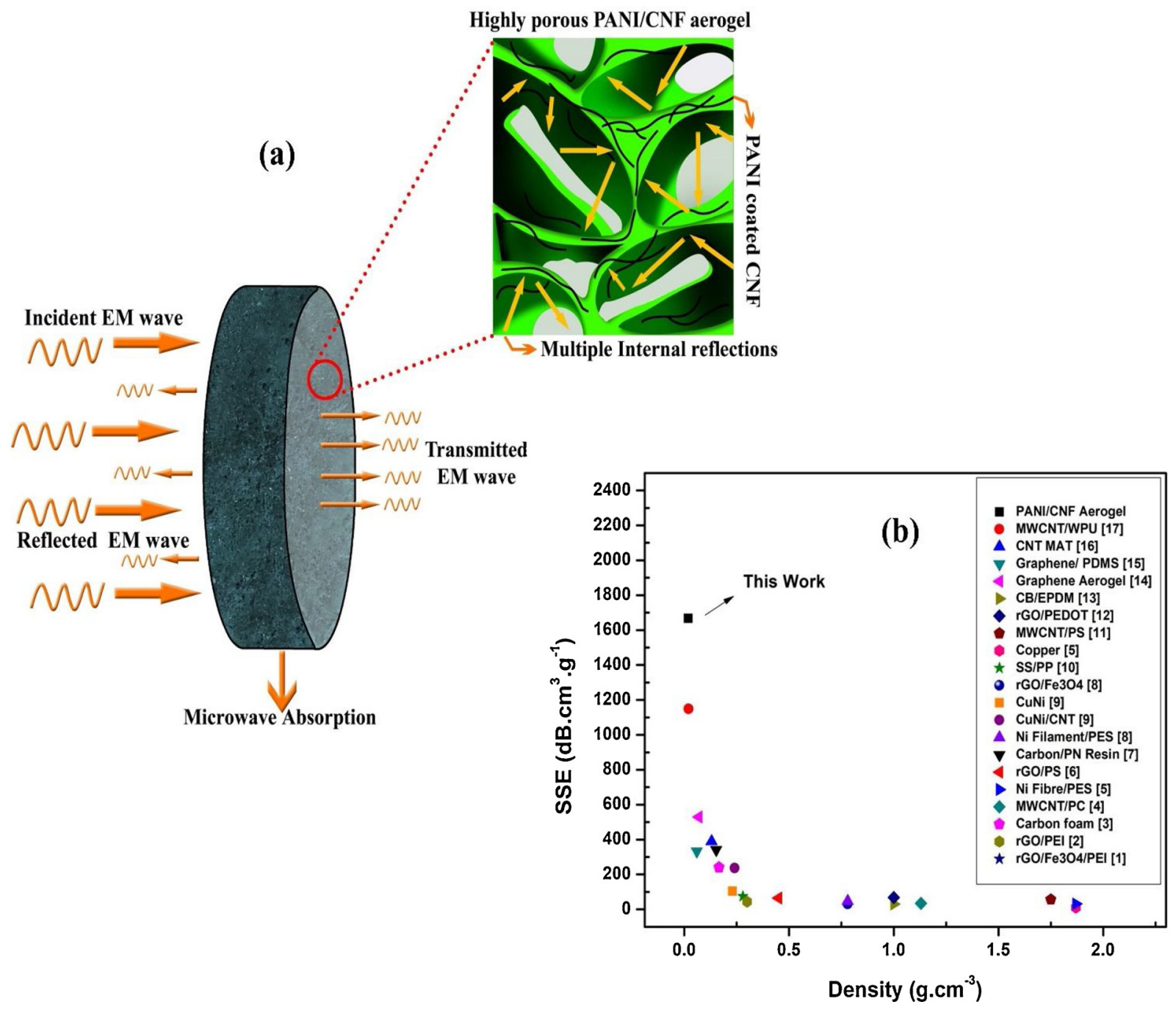

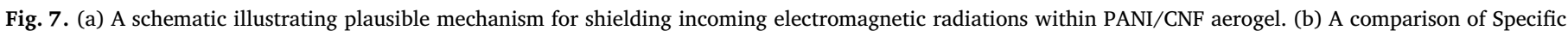

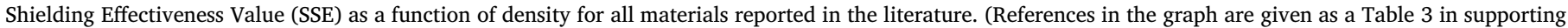
information).

electromagnetic radiation reduces to $1 / \mathrm{e}$ (or $37 \%$ ) of its original strength. This critical thickness is termed as skin depth $(\delta)$ and can be mathematically expressed as below.

$\operatorname{Skin} \operatorname{Depth}(\delta)=\frac{1}{\sqrt{\pi f \mu \sigma}}=-8.68 \frac{t}{S E_{A}}$

where $f$ is frequency, $\mu$ is permeability, $\sigma$ is electrical conductivity $\& \mathrm{t}$ is thickness. The skin effect in PANI/CNF aerogels were investigated to study the depth of electromagnetic wave penetration into the fabricated aerogels. However, it can be observed from the Eq. (8), that skin depth $(\delta)$ of any EMI shield is inversely proportional to shielding by absorption $\left(\mathrm{SE}_{\mathrm{A}}\right)$ and electrical conductivity $(\sigma)$ values. Fig. 8(d) shows variation of skin depth values of all PANI/CNF aerogel composition in the range of $8,2-12.4 \mathrm{GHz}$. It can be clearly seen that as the PANI content increases in the aerogels, the skin depth value shows a decrease from $3.7 \mathrm{~mm}$ to $1.4 \mathrm{~mm}$. This confirms that 1:1 PANI/CNF aerogel showcases a better skin effect when compared to other aerogels with lower concentration of PANI.

The complex permittivity $\left(\varepsilon^{*}=\varepsilon^{\prime}-\mathrm{i} \varepsilon^{\prime \prime}\right)$ of the fabricated aerogels also give some insights into the mechanism of microwave absorption in PANI/CNF aerogels. It is well established that the microwave absorption is closely related with dielectric losses and facilitates enhanced microwave absorption (Agarwal, Kumar, Kumari, \& Dhakate, 2016). The real part of permittivity is related to the amount of polarization occurred in a material/storage of energy whereas the imaginary part of the permittivity is envisaged for the energy losses. These parameters were calculated using the scattering parameters $\left(S_{11} \& S_{21}\right)$ and the standard Nicolson Ross Weir (NRW) method. As per the NRW method, the reflection coefficient of EM wave incident on the shield interface can be expressed as (Nicolson \& Ross, 1970; Weir, 1974)

$\Gamma=\frac{Z-Z_{0}}{Z+Z_{0}}=\left\{\frac{\sqrt{\mu_{r} / \varepsilon_{r}}-1}{\sqrt{\mu_{r} / \varepsilon_{r}}+1}\right\}$

For a shield with fixed thickness (d), the transmission coefficient can be expressed as

$z=e^{-j \omega \sqrt{\mu \varepsilon} d}=e^{\left[-j\left(\frac{\omega}{c}\right) \sqrt{\mu_{r} \varepsilon_{r}} d\right]}$

where, $\mu_{\mathrm{r}}, \varepsilon_{\mathrm{r}}$ are complex permeability, permittivity $\mathrm{Z}_{0}$ is characteristic impedance and $\mathrm{Z}$ is the new characteristic impedance of specimen mounting region.

The sum and difference of the scattering parameters extracted from the VNA can be expressed as

$\mathrm{V}_{1}=\mathrm{S}_{21}+\mathrm{S}_{11}$

$\mathrm{V}_{2}=\mathrm{S}_{21}-\mathrm{S}_{11}$

Form the above equations, reflection coefficient, $\Gamma$ can be calculated 


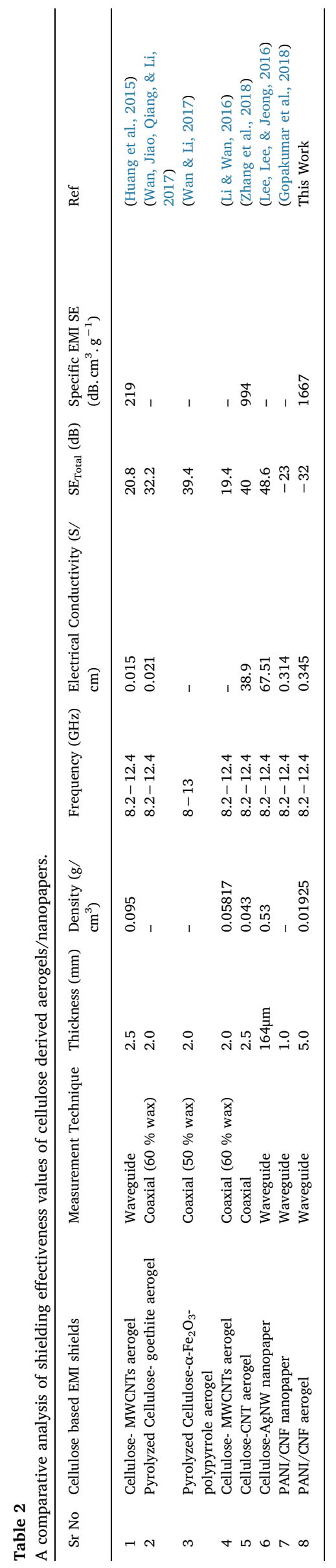

as given below.

$\Gamma=X \pm \sqrt{X^{2}-1}$

where, $X=\frac{1-V_{1} V_{2}}{V_{1}-V_{2}}$ and the appropriate sign is chosen so that $|\Gamma| \leq 1$.

From Eq. (9), it can be defined as,

$\frac{\mu_{r}}{\varepsilon_{r}}=\left[\frac{1+\Gamma}{1-\Gamma}\right]^{2}=C_{1}$

$\mu_{r} \varepsilon_{r}=-\left[\frac{c}{\omega t} \ln \frac{1}{z}\right]^{2}=C_{2}$

Now, the complex permittivity and permeability can be expressed as given below.

$\varepsilon_{r}=\sqrt{\frac{C_{1}}{C_{2}}}$

$\mu_{r}=\sqrt{C_{1} C_{2}}$

The real part, imaginary part of permittivity \& the dielectric loss tangent values of all the fabricated aerogel compositions were plotted as a function of frequency as shown in Fig. 8(a)-(c). It can be clearly seen that at $8.2 \mathrm{GHz}$ the $\varepsilon^{\prime}, \varepsilon^{\prime \prime} \& \operatorname{Tan} \delta_{\varepsilon}$ values of the aerogel compositions were found to be in the order 1:1 > 0.5:1 > 0.2:1. This shows that 1:1 PANI/CNF aerogels showcased large dielectric losses and thus possess enhanced microwave absorption as compared to 0.2:1 and 0.5:1 PANI/CNF aerogels. Table 4 (in supporting Information) provides a comparison of complex permittivity and EMI shielding values of various composite materials.

\subsection{Real time shielding performance of PANI/CNF aerogel with RF detector} circuit

The real time shielding performance of the fabricated PANI/CNF aerogels were studied using a RF detector circuit coupled with an LED output. This powerless device can detect the excess electromagnetic energy emitted from any electronic appliance like mobile phones, microwave ovens etc which operates at different $\mathrm{GHz}$ frequencies. In this work, we made use of a mobile phone to test the real time EMI shielding performance of the fabricated PANI/CNF aerogels. In Fig. 9(a) and (b), the red LED of the RF detector glows as the circuit is brought close a mobile phone in operation. But when we place the fabricated PANI/ CNF aerogels in between the mobile phone and the RF detector, the LED does not glow which indicates that the excess EM energy emanated from the mobile phone is being absorbed by the aerogel. (See Supporting Video S2). The absorbed EM energy will be eventually dissipated as heat energy and hence high power microwave absorption studies of the PANI/CNF aerogels were also performed to elucidate the heat dissipation of PANI/CNF aerogels.

\subsection{High power microwave absorption and ultra-fast heat dissipation studies of PANI/CNF aerogel}

Most of the mobile phones which we use in our day-to-day communication work at a relatively low microwave power (less than $3 \mathrm{~W}$ ). But in order to study the microwave absorption characteristics, PANI/ CNF aerogels were subjected inside a microwave oven (@2.45 GHz and $1000 \mathrm{~W}$ for $6 \mathrm{~s}$ ) which works at a higher power than other microwave devices. Fig. 10(b) show the thermal images of the pristine CNF and PANI/CNF aerogels prior to the microwave exposure. Both the samples were observed to be at ambient conditions. But after the samples were exposed inside a microwave oven for $6 \mathrm{~s}$, the temperature of the PANI/ $\mathrm{CNF}$ aerogels was found to increase up to $102^{\circ} \mathrm{C}$ whereas the neat CNF aerogels still remains at $28^{\circ} \mathrm{C}$. This is due to the fact that PANI molecules can interact with the incoming microwave radiations whereas the neat cellulose nanofibers had no such effect due to its poor electrical conductivity. The thermal images of both aerogels were recorded at 

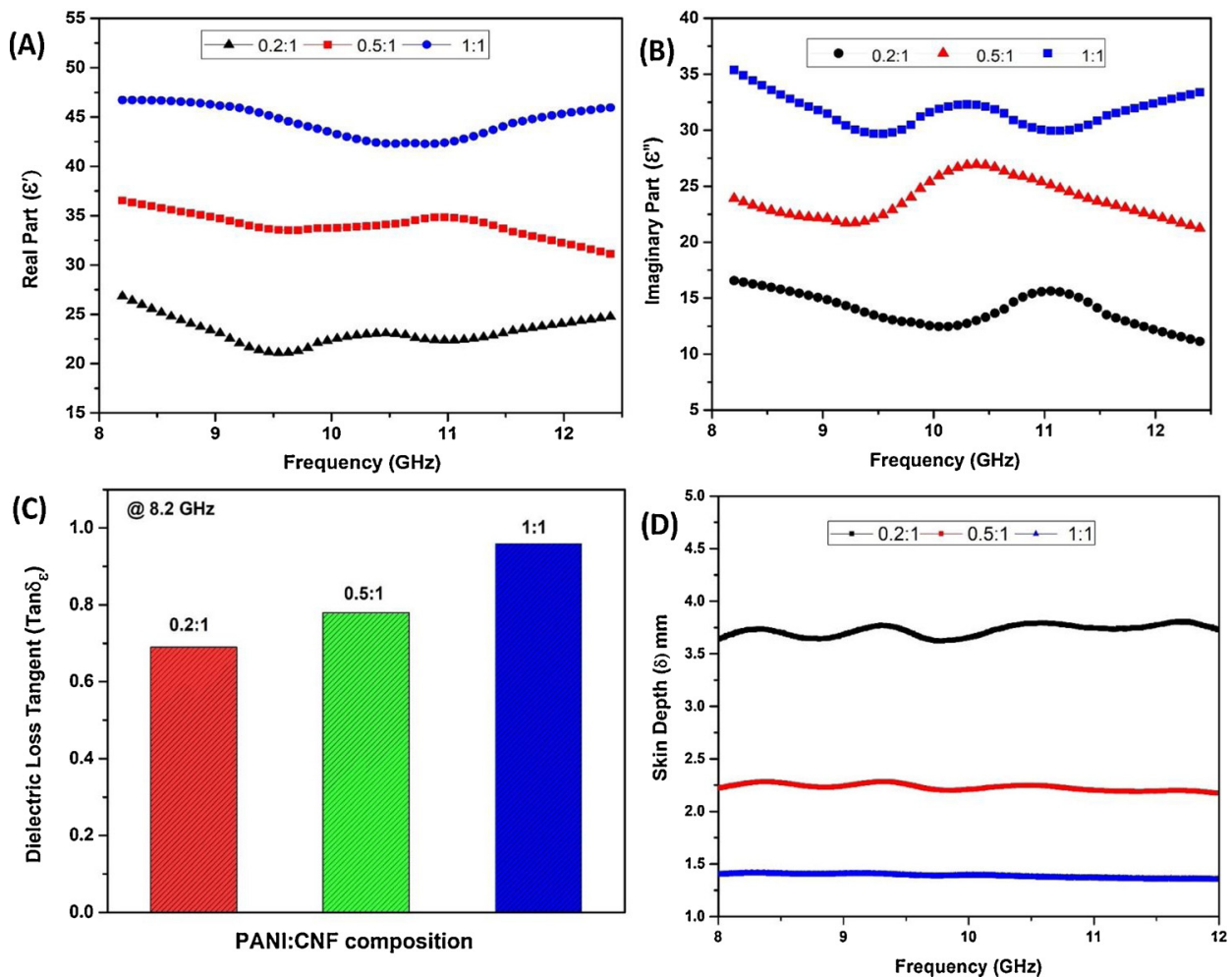

Fig. 8. Frequency dependence of (a) Real part of permittivity ( $\left.\varepsilon^{\prime}\right)$ (b) Imaginary part of permittivity ( $\left.\varepsilon^{\prime \prime}\right)$ (c) Dielectric Loss Tangent (Tan $\delta_{\varepsilon}$ ) @ $8.2 \mathrm{GHz} \&(\mathrm{~d})$ Skin depth $(\delta)$ of all PANI/CNF aerogel compositions.

every $5 \mathrm{~s}$ (see Fig. 10(C-L)) and the heat energy emanated due to the microwave absorption was dissipated to the surrounding air in a span of $45 \mathrm{~s}$ (See Supporting Video S3). According to the Newton's law of cooling, the rate of heat loss from a system is governed by the following equation.

$\frac{d Q}{d t}=h A\left(T_{S}-T_{\infty}\right)$

Where, $\mathrm{Q}$ is the thermal energy, $\mathrm{h}$ is the heat transfer coefficient, $\mathrm{A}$ is the surface area, $T_{S}$ is surface temperature and $T_{\infty}$ is the temperature of the surroundings. In the case of PANI/CNF aerogels there was an exponentially decreasing heat dissipation profile after exposure to microwave radiation for $6 \mathrm{~s}$ as shown in Fig. 10 (see supporting information). This ultra-fast heat dissipation behavior can be envisaged due to the high porosity and specific surface area of the fabricated aerogels (Kim, Oh, \& Islam, 2013). The uniform coating of polyaniline in the PANI/CNF aerogels interacts with the incident microwave radiation and gets excited to a higher energy state due to the molecular vibrations in the PANI and eventually comes back to the ground sate due to vibrational relaxations thus liberating heat energy (Javadi,

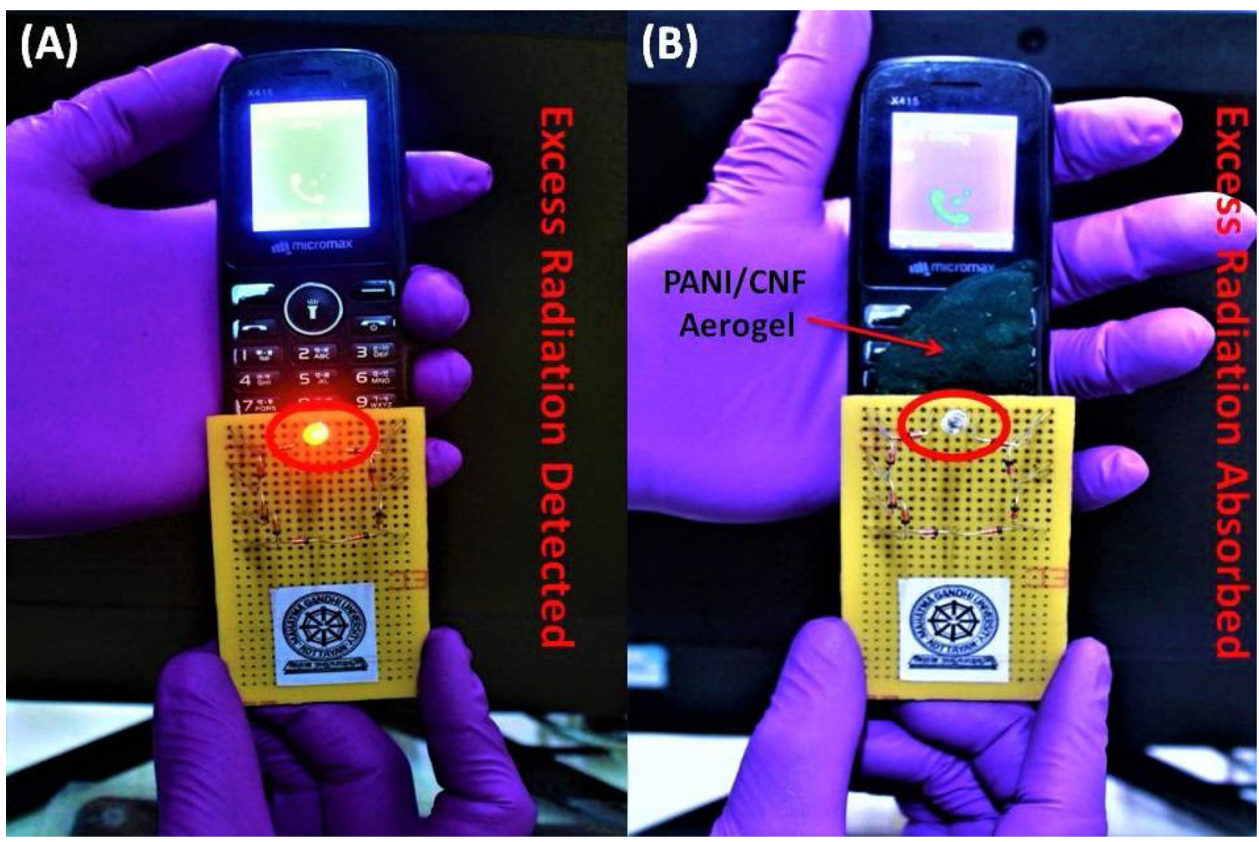

Fig. 9. (A) An RF detector circuit detecting the leakage of undesired EM radiations emitted out from a mobile phone while in use (Red LED glows); (B) An intuitive evidence that the fabricated PANI/CNF aerogel absorbs the excess EM radiations when placed above the mobile phone (LED does not glow). (For interpretation of the references to colour in this figure legend, the reader is referred to the web version of this article). 

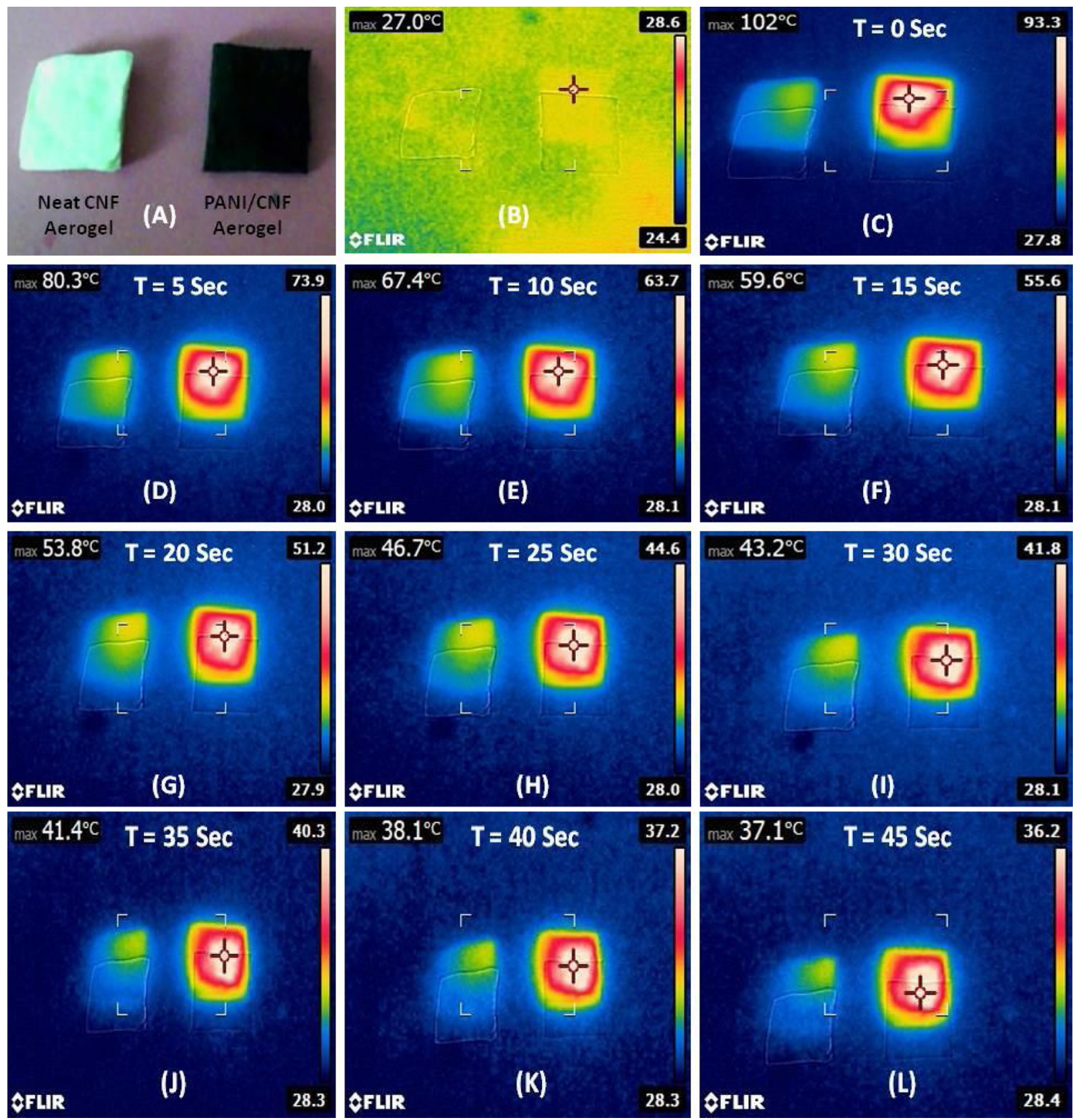

Fig. 10. (A) Digital images of neat CNF (left) and PANI/CNF aerogels (right) (B) The thermal images of neat CNF and PANI/CNF aerogels before the microwave test. (C-L) Thermal images taken after irradiating neat CNF and PANI/CNF aerogels inside a microwave oven (@2.45 GHz \& $1000 \mathrm{~W}$ ) for $6 \mathrm{~s}$. (Images taken at time interval of 5 s).

Cromack, MacDiarmid, \& Epstein, 1989). This heat energy was then radiated to the surrounding air pockets via convection mode of heat transfer to facilitate an ultra-fast heat dissipation behavior in the PANI/ $\mathrm{CNF}$ aerogels. In addition, from the thermogravimetric analysis, PANI/ $\mathrm{CNF}$ aerogels were found to be stable up to $200^{\circ} \mathrm{C}$ without much degradation which infers that the demonstrated aerogels are also quite stable and suitable for use in high power microwave devices.

\section{Conclusion}

A series of lighweight and 3D porous conductive aerogels derived from polyaniline achored cellulose nanofibers were fabricated via an efficient and environmentally friendly stategy. The fabricted PANI/CNF aerogels exhibited densities as low as $0.01925 \mathrm{~g} / \mathrm{cc}$ with a high porosity of $98.6 \%$. Moreover, morphological analysis infers that the fabricated
PANI/CNF aerogels showcased a continuous porous web like conductive structures. Furthermore, the formation of a well interconnected coating of polyaniline on cellulose nanofibers resulted in a higher electrical conductivity value of $0.345 \mathrm{~S} / \mathrm{cm}$ in PANI/CNF aerogels. This was attributed to the strong inter molecular hydrogen between cellulose nanofibers and PANI which was confirmed from FTIR studies. The PANI/CNF aerogels with 1:1 ratio exhibited a maximum EMI shielding effectiveness of ca. $-32 \mathrm{~dB}$ (> $99.9 \%$ attenuation) @ $8.2 \mathrm{GHz}$ and $5.0 \mathrm{~mm}$ thickness. It is also noteworthy that PANI/CNF aerogels attenuated the incoming microwave radiations primarily via absorption dominant mechanism (ca. $95 \%$ ) and only minimal amount of EM radiations were reflected (ca. $5 \%$ ). The heat dissipation study reveals that the PANI/CNF aerogels had a strong ability to absorb high power microwave radiations when placed in a microwave oven and also possess ultra-fast heat dissipation ability. These light weight aerogels also 
exhibited a specific EMI shielding effectiveness of $\sim 1667 \mathrm{~dB} \cdot \mathrm{g}^{-1} . \mathrm{cm}^{-3}$ in $8.2-12.4 \mathrm{GHz}$ (X Band) region. These results portray that PANI/CNF aerogels open up new horizons for the development of sustainable and green microwave attenuators for portable electronic devices.

\section{AUTHORSHIP STATEMENT}

1. Avinash R Pai - Conception and design of study; acquisition, analysis and/or interpretation of data, drafting the manuscript.

2. Binumol T- acquisition of data

3. Deepu A. Gopakumar- analysis and/or interpretation of data

4. Daniel Pasquini- Validation.

5. Bastien Seantier- Writing - review \& editing

6. Nandakumar Kalarikkal- Visualization

7. Sabu Thomas- Supervision

\section{Declaration of Competing Interest}

The authors declare no conflict of interest.

\section{Acknowledgement}

ARP greatefully acknowledges the financial support from the Ministry of Electronics and IT (MeitY), Digital India Corporation, (Formerly Medialab Asia), New Delhi, India provided under the Visvesvaraya Ph.D Scheme (Ref No: PhD-MLA/4(58)/2015-16).

\section{Appendix A. Supplementary data}

Supplementary material related to this article can be found, in the online version, at doi:https://doi.org/10.1016/j.carbpol.2020.116663.

\section{References}

Agarwal, P. R., Kumar, R., Kumari, S., \& Dhakate, S. R. (2016). Three-dimensional and highly ordered porous carbon-MnO2 composite foam for excellent electromagnetic interference shielding efficiency. RSC Advances, 6(103), 100713-100722. https:// doi.org/10.1039/c6ra23127f.

Al-Saleh, M. H., \& Sundararaj, U. (2009). Electromagnetic interference shielding mechanisms of CNT/polymer composites. Carbon, 47(7), 1738-1746. https://doi.org/ 10.1016/j.carbon.2009.02.030.

Cao, W. T., Chen, F. F., Zhu, Y. J., Zhang, Y. G., Jiang, Y. Y., Ma, M. G., \& Chen, F. (2018). Binary strengthening and toughening of MXene/Cellulose nanofiber composite paper with nacre-inspired structure and superior electromagnetic interference shielding properties. ACS Nano, 12(5), 4583-4593. https://doi.org/10.1021/acsnano.8b00997 research-article.

Chen, Y., Zhang, H. B., Yang, Y., Wang, M., Cao, A., \& Yu, Z. Z. (2016). High-performance epoxy nanocomposites reinforced with three-dimensional carbon nanotube sponge for electromagnetic interference shielding. Advanced Functional Materials, 26(3), 447-455. https://doi.org/10.1002/adfm.201503782.

Dalal, J., Lather, S., Gupta, A., Tripathi, R., Maan, A. S., Singh, K., \& Ohlan, A. (2019). Reduced graphene oxide functionalized strontium ferrite in poly(3,4-ethylenedioxythiophene) conducting network: a high-performance EMI shielding material. Advanced Materials \& TechnologiesArticle 1900023. https://doi.org/10.1002/admt. 201900023.

Dar, M. A., Majid, K., Hanief Najar, M., Kotnala, R. K., Shah, J., Dhawan, S. K., \& Farukh, M. (2017). Surfactant-assisted synthesis of polythiophene/Ni0.5Zn0.5Fe2-: XCexO4 ferrite composites: Study of structural, dielectric and magnetic properties for EMIshielding applications. Journal of the Chemical Society Faraday Transactions, 19(16), 10629-10643. https://doi.org/10.1039/c7cp00414a.

Eftekhari, A., Li, L., \& Yang, Y. (2017). Polyaniline supercapacitors. Journal of Power Sources, 347, 86-107.

Erogul, O., Oztas, E., Yildirim, I., Kir, T., Aydur, E., Komesli, G., ... Peker, A. F. (2006) Effects of electromagnetic radiation from a cellular phone on human sperm motility: An in vitro study. Archives of Medical Research, 37(7), 840-843. https://doi.org/10. 1016/j.arcmed.2006.05.003.

French, A. D. (2014). Idealized powder diffraction patterns for cellulose polymorphs. Cellulose, 21(2), 885-896. https://doi.org/10.1007/s10570-013-0030-4.

Fugetsu, B., Sano, E., Sunada, M., Sambongi, Y., Shibuya, T., Wang, X., \& Hiraki, T. (2008). Electrical conductivity and electromagnetic interference shielding efficiency of carbon nanotube/cellulose composite paper. Carbon, 46(9), 1256-1258.

Gopakumar, D. A., Pai, A. R., Pottathara, Y. B., Pasquini, D., Carlos De Morais, L., Luke, M., ... Thomas, S. (2018). Cellulose nanofiber-based polyaniline flexible papers as sustainable microwave absorbers in the X-Band. ACS Applied Materials \& Interfaces, 10(23), 20032-20043. https://doi.org/10.1021/acsami.8b04549.
Guo, J., Song, H., Liu, H., Luo, C., Ren, Y., Ding, T., ... Guo, Z. (2017). Polypyrroleinterface-functionalized nano-magnetite epoxy nanocomposites as electromagnetic wave absorbers with enhanced flame retardancy. Journal of Materials Chemistry C, 5(22), 5334-5344. https://doi.org/10.1039/c7tc01502j.

Hu, W., Chen, S., Yang, Z., Liu, L., \& Wang, H. (2011). Flexible electrically conductive nanocomposite membrane based on bacterial cellulose and polyaniline. The Journal of Physical Chemistry B, 115(26), 8453-8457. https://doi.org/10.1021/jp204422v.

Huang, H. D., Liu, C. Y., Zhou, D., Jiang, X., Zhong, G. J., Yan, D. X., \& Li, Z. M. (2015). Cellulose composite aerogel for highly efficient electromagnetic interference shielding. Journal of Materials Chemistry A, 3(9), 4983-4991. https://doi.org/10. 1039/c4ta05998k.

Javadi, H. H. S., Cromack, K. R., MacDiarmid, A. G., \& Epstein, A. J. (1989). Microwave transport in the emeraldine form of polyaniline. Physical Review B, 39(6), 3579-3584.

Joseph, N., Varghese, J., \& Sebastian, M. T. (2015). Self assembled polyaniline nanofibers with enhanced electromagnetic shielding properties. RSC Advances, 5(26), 20459-20466. https://doi.org/10.1039/b000000x.

Kaur, A., Ishpal, \& Dhawan, S. K. (2012). Tuning of EMI shielding properties of polypyrrole nanoparticles with surfactant concentration. Synthetic Metals, 162(15-16), 1471-1477. https://doi.org/10.1016/j.synthmet.2012.05.012.

Khadieva, A. I., Gorbachuk, V. V., Evtugyn, G. A., Belyakova, S. V., Latypov, R. R., Drobyshev, S. V., \& Stoikov, I. I. (2019). Phenyliminophenothiazine based self-organization of polyaniline nanowires and application as redox probe in electrochemical sensors. Scientific Reports, 9(1), 1-10. https://doi.org/10.1038/s41598-01836937-5.

Kim, K. H., Oh, Y., \& Islam, M. F. (2013). Mechanical and thermal management characteristics of ultrahigh surface area single-walled carbon nanotube aerogels. Advanced Functional Materials, 23(3), 377-383. https://doi.org/10.1002/adfm. 201201055.

Kobayashi, Y., Saito, T., \& Isogai, A. (2014). Aerogels with 3D ordered nanofiber skeletons of liquid-crystalline nanocellulose derivatives as tough and transparent insulators. Angewandte Chemie (International Ed in English), 53(39), 10394-10397. https://doi org/10.1002/anie.201405123.

Kulkarni, G., Kandesar, P., Velhal, N., Phadtare, V., Jatratkar, A., Shinde, S. K., ... Puri, V. (2019). Exceptional electromagnetic interference shielding and microwave absorption properties of room temperature synthesized polythiophene thin films with double negative characteristics (DNG) in the Ku-band region. Chemical Engineering Journal, 355(August 2018), 196-207. https://doi.org/10.1016/j.cej.2018.08.114.

Lee, T. W., Lee, S. E., \& Jeong, Y. G. (2016). Highly effective electromagnetic interference shielding materials based on silver Nanowire/Cellulose papers. ACS Applied Materials \& Interfaces, 8(20), 13123-13132. https://doi.org/10.1021/acsami.6b02218.

Li, J., \& Wan, C. (2016). Cellulose aerogels decorated with multi-walled carbon nanotubes: Preparation, characterization, and application for electromagnetic interference shielding. Frontiers of Agricultural Science and Engineering, 2(4), 341-346. https://doi. org $/ 10.15302 /$ J-FASE-2015082.

Li, W., Gao, F., Wang, X., Zhang, N., \& Ma, M. (2016). Strong and robust polyanilinebased supramolecular hydrogels for flexible supercapacitors. Angewandte Chemie International Edition, 55(32), 9196-9201. https://doi.org/10.1002/anie.201603417.

Luo, H., Dong, J., Zhang, Y., Li, G., Guo, R., Zuo, G., ... Wan, Y. (2018). Constructing 3D bacterial cellulose/graphene/polyaniline nanocomposites by novel layer-by-layer in situ culture toward mechanically robust and highly flexible freestanding electrodes for supercapacitors. Chemical Engineering Journal, 334, 1148-1158. https://doi.org/ 10.1016/j.cej.2017.11.065.

Luo, S. J., Zhang, P., Mei, Y. A., Chang, J. B., \& Yan, H. (2016). Electromagnetic interference shielding properties of PEDOT/PSS-halloysite nanotube (HNTs) hybrid films. Journal of Applied Polymer Science, 133(47), 1-5. https://doi.org/10.1002/app. 44242.

Mohsennia, M., Bidgoli, M. M., Boroumand, F. A., \& Nia, A. M. (2015). Electrically conductive polyaniline as hole-injection layer for MEH-PPV:BT based polymer light emitting diodes. Materials Science \& Engineering B, Solid-state Materials for Advanced Technology, 197, 25-30. https://doi.org/10.1016/j.mseb.2015.03.003.

Mondal, S., Ganguly, S., Das, P., Bhawal, P., Das, T. K., Nayak, L., ... Das, N. C. (2017). High-performance carbon nanofiber coated cellulose filter paper for electromagnetic interference shielding. Cellulose, 24(11), 5117-5131. https://doi.org/10.1007/ s10570-017-1441-4.

Nicolson, A. M., \& Ross, G. F. (1970). Measurement of the intrinsic properties of materials by time-domain techniques. IEEE Transactions on Instrumentation and Measurement, 19(4), 377-382. https://doi.org/10.1109/TIM.1970.4313932.

Park, H., Jeong, Y. R., Yun, J., Hong, S. Y., Jin, S., Lee, S. J., ... Ha, J. S. (2015). Stretchable array of highly sensitive pressure sensors consisting of polyaniline nanofibers and Au-Coated polydimethylsiloxane micropillars. ACS Nano, 9(10), 9974-9985. https://doi.org/10.1021/acsnano.5b03510.

Shen, B., Zhai, W., \& Zheng, W. (2014). Ultrathin flexible graphene film: An excellent thermal conducting material with efficient EMI shielding. Advanced Functional Materials, 24(28), 4542-4548. https://doi.org/10.1002/adfm.201400079.

Shi, Z., Zang, S., Jiang, F., Huang, L., Lu, D., Ma, Y., \& Yang, G. (2012). In situ nanoassembly of bacterial cellulose-polyaniline composites. RSC Advances, 2(3), 1040-1046. https://doi.org/10.1039/c1ra00719j.

Sun, R., Zhang, H. B., Liu, J., Xie, X., Yang, R., Li, Y., ... Yu, Z. Z. (2017). Highly conductive transition metal Carbide/Carbonitride(MXene)@polystyrene nanocomposites fabricated by electrostatic assembly for highly efficient electromagnetic interference shielding. Advanced Functional Materials, 27(45), 1-11. https://doi.org/10.1002/ adfm.201702807.

Tian, Y., Zhang, X., Dou, S., Zhang, L., Zhang, H., \& Lv, H. (2017). Solar energy materials and solar cells a comprehensive study of electrochromic device with variable infrared emissivity based on polyaniline conducting polymer. Solar Energy Materials and Solar Cells, 170, 120-126. https://doi.org/10.1016/j.solmat.2017.05.053. 
Wan, C., \& Li, J. (2016). Graphene oxide/cellulose aerogels nanocomposite: Preparation, pyrolysis, and application for electromagnetic interference shielding. Carbohydrate Polymers, 150, 172-179. https://doi.org/10.1016/j.carbpol.2016.05.051.

Wan, C., \& Li, J. (2017). Synthesis and electromagnetic interference shielding of cellulosederived carbon aerogels functionalized with $\alpha-\mathrm{Fe} 2 \mathrm{O} 3$ and polypyrrole. Carbohydrate Polymers, 161, 158-165. https://doi.org/10.1016/j.carbpol.2017.01.003.

Wan, C., Jiao, Y., Qiang, T., \& Li, J. (2017). Cellulose-derived carbon aerogels supported goethite $(\alpha-\mathrm{FeOOH})$ nanoneedles and nanoflowers for electromagnetic interference shielding. Carbohydrate Polymers, 156, 427-434. https://doi.org/10.1016/j.carbpol. 2016.09.028.

Wang, Q. W., Zhang, H. B., Liu, J., Zhao, S., Xie, X., Liu, L., ... Yu, Z. Z. (2019). Multifunctional and water-resistant MXene-Decorated polyester textiles with outstanding electromagnetic interference shielding and joule heating performances. Advanced Functional Materials, 29(7), 1-10. https://doi.org/10.1002/adfm. 201806819.

Weir, W. B. (1974). Automatic measurement of complex dielectric constant and permeability at microwave frequencies. Proceedings of the IEEE, 62(1), 33-36. https://doi org/10.1109/PROC.1974.9382.

Weng, G. M., Li, J., Alhabeb, M., Karpovich, C., Wang, H., Lipton, J., ... Taylor, A. D. (2018). Layer-by-layer assembly of cross-functional semi-transparent MXene-carbon nanotubes composite films for next-generation electromagnetic interference shielding. Advanced Functional Materials, 28(44), 1-9. https://doi.org/10.1002/adfm. 201803360.

Wu, X., Zhang, W., Wang, Q., Wang, Y., Yan, H., \& Chen, W. (2016). Hydrogen bonding of graphene/polyaniline composites film for solid electrochromic devices. Synthetic Metals, 212, 1-11.

Wu, F., Xie, A., Sun, M., Wang, Y., \& Wang, M. (2015). Reduced graphene oxide (RGO) modified spongelike polypyrrole (PPy) aerogel for excellent electromagnetic absorption. Journal of Materials Chemistry A, 3(27), 14358-14369. https://doi.org/10. 1039/c5ta01577d.

Wu, X., Lu, C., Xu, H., Zhang, X., \& Zhou, Z. (2014). Biotemplate synthesis of polyaniline@cellulose nanowhiskers/natural rubber nanocomposites with 3D hierarchical multiscale structure and improved electrical conductivity. ACS Applied Materials \& Interfaces, 6(23), 21078-21085. https://doi.org/10.1021/am505924z.

Yan, D. X., Pang, H., Li, B., Vajtai, R., Xu, L., Ren, P. G., ... Li, Z. M. (2015). Structured reduced graphene oxide/polymer composites for ultra-efficient electromagnetic interference shielding. Advanced Functional Materials, 25(4), 559-566. https://doi.org/ 10.1002/adfm.201403809.
Yang, Y., Gupta, M. C., Dudley, K. L., \& Lawrence, R. W. (2005). Novel carbon nanotube Polystyrene foam composites for electromagnetic interference shielding. Nano Letters, 5(11), 2131-2134. https://doi.org/10.1021/nl051375r.

Yu, H., Chen, P., Chen, W., \& Liu, Y. (2014). Effect of cellulose nanofibers on induced polymerization of aniline and formation of nanostructured conducting composite. Cellulose, 21(3), 1757-1767. https://doi.org/10.1007/s10570-014-0189-3.

Zamanian, B. A., \& Hardiman, C. (2005). Electromagnetic radiation and human health: A review of sources and effects. High Frequency Electronics, 4(3), 16-26 Retrieved from http://gnusha.org/ nmz787/biological radio research/Electromagnetic Radiation and Human Health_A Review of Sources and Effects_Fluor Corporation.pdf.

Zeng, Z., Jin, H., Chen, M., Li, W., Zhou, L., \& Zhang, Z. (2016). Lightweight and anisotropic porous MWCNT/WPU composites for ultrahigh performance electromagnetic interference shielding. Advanced Functional Materials, 26(2), 303-310. https://doi.org/10.1002/adfm.201503579.

Zhang, L. Q., Yang, S. G., Li, L., Yang, B., Huang, H. D., Yan, D. X., ... Li, Z. M. (2018). Ultralight cellulose porous composites with manipulated porous structure and carbon nanotube distribution for promising electromagnetic interference shielding. ACS Applied Materials \& Interfaces, 10(46), 40156-40167. https://doi.org/10.1021/ acsami.8b14738.

Zhang, Y., Yang, Z., Yu, Y., Wen, B., Liu, Y., \& Qiu, M. (2019). Tunable Electromagnetic Interference Shielding Ability in a One-Dimensional Bagasse Fiber/Polyaniline Heterostructure. ACS Applied Polymer Materials, 1(4), 737-745. https://doi.org/10 1021/acsapm.8b00025.

Zhao, S., Zhang, H. B., Luo, J. Q., Wang, Q. W., Xu, B., Hong, S., \& Yu, Z. Z. (2018). Highly electrically conductive three-dimensional Ti3C2Tx MXene/Reduced graphene oxide hybrid aerogels with excellent electromagnetic interference shielding performances. ACS Nano, 12(11), 11193-11202. https://doi.org/10.1021/acsnano.8b05739.

Zhou, S., Liu, P., Wang, M., Zhao, H., Yang, J., \& Xu, F. (2016). Sustainable, reusable, and superhydrophobic aerogels from microfibrillated cellulose for highly effective Oil/ Water separation. ACS Sustainable Chemistry \& Engineering, 4(12), 6409-6416. https://doi.org/10.1021/acssuschemeng.6b01075.

Zhou, Z., Zhang, X., Lu, C., Lan, L., \& Yuan, G. (2014). Polyaniline-decorated cellulose aerogel nanocomposite with strong interfacial adhesion and enhanced photocatalytic activity. RSC Advances, 4(18), 8966-8972. https://doi.org/10.1039/c3ra46441e.

Zhu, W., Chen, X. L., Chang, J., Yu, R. M., Li, H., Liang, D., ... Lu, C. Z. (2018). Doped polyaniline-hybridized tungsten oxide nanocrystals as hole injection layers for efficient organic light-emitting diodes. Journal of Materials Chemistry C, 6(27), 7242-7248. https://doi.org/10.1039/c8tc01005f. 